Revue musicale OICRM

\title{
Musicologie pratique à l'ère des réseaux sociaux. Le cas des notes de programme numériques de l'osm au sein du paysage musical francophone
}

\section{Justin Bernard}

Volume 6, numéro 2, 2020

Musicologie numérique. Vers de nouvelles frontières disciplinaires

URI : https://id.erudit.org/iderudit/1068386ar

DOI : https://doi.org/10.7202/1068386ar

Aller au sommaire du numéro

Éditeur(s)

Observatoire interdisciplinaire de création et recherche en musique (OICRM)

ISSN

2368-7061 (numérique)

Découvrir la revue

Citer cet article

Bernard, J. (2020). Musicologie pratique à l'ère des réseaux sociaux. Le cas des notes de programme numériques de l'osm au sein du paysage musical francophone. Revue musicale OICRM, 6(2), 73-106.

https://doi.org/10.7202/1068386ar
Résumé de l'article

Depuis leur émergence au milieu du XIX ${ }^{\mathrm{e}}$ siècle, les notes de programme n'ont eu de cesse de s'adresser au public de concerts. Les besoins sont multiples, voire même divergents : donner des outils de compréhension intelligibles aux novices et en même temps susciter la curiosité d'auditeurs déjà initiés. À l'ère d'Internet et des réseaux sociaux, ces outils de médiation, qui relèvent de ce que l'on pourrait appeler une musicologie « pratique ", sont plus que jamais en évolution. Ils offrent de réelles avancées en termes de contenu audio et vidéo, mais suscitent, par ailleurs, des interrogations quant au rôle que les orchestres symphoniques, notamment, veulent bien leur faire jouer. Dans cet article, nous tenterons de répondre à cette nouvelle réalité à travers une multitude d'exemples concrets de médiation, dont certains ont été réalisés dans le cadre d'un projet que nous avons mené en partenariat avec l'Orchestre symphonique de Montréal. 


\title{
Musicologie pratique à l'ère des réseaux sociaux. Le cas des notes de programme numériques de l'osm au sein du paysage musical francophone
}

Justin Bernard

Résumé

Depuis leur émergence au milieu du $\mathrm{XIX}^{\mathrm{e}}$ siècle, les notes de programme n'ont eu de cesse de s'adresser au public de concerts. Les besoins sont multiples, voire même divergents : donner des outils de compréhension intelligibles aux novices et en même temps susciter la curiosité d'auditeurs déjà initiés. À l'ère d'Internet et des réseaux sociaux, ces outils de médiation, qui relèvent de ce que l'on pourrait appeler une musicologie "pratique ", sont plus que jamais en évolution. Ils offrent de réelles avancées en termes de contenu audio et vidéo, mais suscitent, par ailleurs, des interrogations quant au rôle que les orchestres symphoniques, notamment, veulent bien leur faire jouer. Dans cet article, nous tenterons de répondre à cette nouvelle réalité à travers une multitude d'exemples concrets de médiation, dont certains ont été réalisés dans le cadre d'un projet que nous avons mené en partenariat avec l'Orchestre symphonique de Montréal.

Mots clés : guides d'écoute; Internet ; médiation ; notes de programme ; réseaux sociaux.

\begin{abstract}
Since their appearance in the mid-nineteenth century, program notes, sometimes containing detailed information on the works that are to be performed, have continued to meet a wide range of audiences needs during concerts, such as: providing intelligible tools for laymen and laywomen to decipher while arousing the curiosity of well-informed listeners. In the age of Internet and Social media, these music appreciation tools, which fall within what we may call "practical" musicology, are evolving more than ever. They offer significant improvement in terms of audio and visual content, but raise some questions concerning the actual role that symphonic orchestras in particular are willing to see them play. In this article we will try to respond to this new reality through a series of concrete examples of music appreciation tools, including some which were produced in the context of a project we conducted in partnership with the Orchestre symphonique de Montréal.
\end{abstract}

Keywords: Internet; listening guides; music appreciation; program notes; social media. 
Au-delà des différents domaines de spécialisation en musicologie (histoire de la musique, psychologie de la musique, ethnomusicologie, etc.), il semble y avoir deux manières fondamentales d'aborder la discipline dans son ensemble ${ }^{1}$. La première est une musicologie qui relève de recherches approfondies et de théories à long terme. Celle-ci est doublement imprégnée par l'idée de postérité. À travers des ouvrages ou une série d'articles, le musicologue cherche non seulement à garantir la postérité d'une musique ou d'une communauté, quelles qu'elle soient, mais également à assurer sa propre postérité, en s'adressant aussi bien à un lecteur contemporain qu'à un lecteur futur. La musicologie " pratique ", quant à elle, se rattache davantage à l'idée d'actualité. À travers des textes plus courts et plus ponctuels, le musicologue ${ }^{2}$ s'adresse surtout aux lecteurs contemporains, aux abonnés d'une revue, aux publics de concert $^{3}$.

Dans cet article, nous nous intéresserons à cette seconde approche et, en particulier, à un certain type d'écrits, propre au concert, qui permet au musicologue d'exercer pleinement son champ d'expertise. Nous voulons ici parler de la note de programme. Plutôt que de clore l'expérience de concert comme le fait la critique musicale, la note de programme servira à préparer, voire à guider l'auditeur dans son écoute à venir. Commandée pour un concert ou une série de concerts, elle remplit une fonction spécifique et s'adresse à un public plus large, non seulement aux mélomanes, aux acteurs du milieu musical et aux universitaires, mais aussi aux moins initiés. En cela, la note de programme relève autant d'une musicologie pratique que d'une recherche (musicologique) appliquée, en complément d'une recherche fondamentale qui évolue au sein du milieu académique à travers notamment les centres de recherches, les colloques et les publications d'ouvrages ou d'articles scientifiques ${ }^{4}$.

Avec l'aide des musicologues praticiens, les orchestres symphoniques continuent de produire des notes de programme, et ce, depuis le XIX ${ }^{e}$ siècle. L'apparition de l'ordinateur et des nouvelles technologies ont toutefois révolutionné notre rapport à

1 Cet article reprend, en partie, des éléments de la thèse de doctorat de Justin Bernard, intitulée Notes de programme. Une histoire, des pratiques et de nouveaux usages numériques (Bernard 2019).

2 Dans le cadre de notre réflexion, nous ne ferons pas de distinction particulière entre musicologues et musicographes. Certes, un musicographe ne possède pas les mêmes qualifications qu'un musicologue et son approche de l'écriture demeure volontairement moins scientifique - prises de position assumées, absence de références, absence de bibliographie, pour ne donner que ces exemples-là -, mais nous nous intéressons ici plutôt à la démarche commune, celle de transmettre un ensemble de connaissances musicales à un vaste lectorat.

3 Il n'est cependant pas exclu que ces textes puissent être lus et relus dans un avenir lointain.

4 Le milieu académique n'est pas le seul réservoir de chercheurs en musicologie. Sans être nécessairement rattachés à une institution d'enseignement, des musicologues ou musicographes entreprennent, à titre personnel, l'écriture d'ouvrages détaillés sur un sujet lié à l'histoire de la musique ou à la musique d'aujourd'hui. C'est notamment le cas d'Alex Ross, critique musical au journal The New Yorker, qui est l'auteur d'un essai documenté sur la musique du $\mathrm{xx}^{\mathrm{e}}$ siècle : The Rest is Noise. Listening to the Twentieth Century (Ross 2008). À noter qu'il est, aujourd'hui, assez rare que soit publié un ouvrage d'envergure comme celui-ci et dont l'auteur soit une personne extérieure au monde de la recherche. En revanche, au $\mathrm{XIX}^{\mathrm{e}}$ siècle, à l'époque où la musicologie ne s'était pas encore établie dans la plupart des pays d'Europe, beaucoup d'écrits sur la musique étaient le fruit de musicographes. 
l'information. À l'ère d'Internet et des réseaux sociaux, nous assistons aujourd'hui à de nouveaux modes de communication entre les orchestres et leurs publics ${ }^{5}$. Les points de contact et les sources d'information se multiplient. Pour connaître le calendrier des concerts, les mélomanes peuvent désormais consulter librement le site web officiel de ces institutions musicales ; beaucoup d'entre eux s'abonnent à l'infolettre qu'ils reçoivent ensuite par courriel. À cela s'ajoutent les interactions multiples sur Facebook, sur Twitter ou sur YouTube, plateformes web où les internautes peuvent « aimer » (liker) une publication, une vidéo, laisser un commentaire.

Les réseaux sociaux constituent un formidable outil de promotion pour les orchestres qui deviennent ainsi leur propre média et gèrent leur propre service de communication. Pour continuer à susciter l'intérêt - et augmenter leur nombre d'abonnés sur le web -, les orchestres proposent une diversité de contenus. Ils publient en amont de l'évènement ciblé (ex. : concours de billets, lien vers des articles de presse, lien audio/vidéo vers des entrevues, présentation des artistes au programme), mais aussi en aval (lien vers une critique du concert, retour sur l'évènement, photos-souvenirs $\mathrm{du}$ concert, etc.). Parmi les contenus en amont, on retrouve notamment la liste des œuvres au programme, égrenée par les orchestres au fil de la saison. Se pose alors la question de savoir comment parler des œuvres et des compositeurs à un public très diversifié, qui n'a pas nécessairement les mêmes bases de compréhension.

Dans cet article, nous aborderons les diverses méthodes employées aujourd'hui pour décrire et raconter adéquatement la musique ; celle-là même qui sera entendue dans le cadre des concerts publics. Nous nous intéresserons en particulier aux notes de programme, comme modèles de communication écrite, et verrons en quoi celles-ci peuvent prendre de nouvelles formes à l'ère du numérique.

Déjà au début du $\mathrm{Xx}^{\mathrm{e}}$ siècle, les orchestres prennent conscience du fait que les notes de programme ne représentent qu'une partie de la préparation de l'auditeur avant le concert. D'autres dispositifs, qui dépassent le cadre strictement écrit d'une note de programme, sont à mettre à profit pour raffermir le lien avec l'œuvre musicale. Dans son article « Towards a History of the Programme Note », Christina Bashford explique :

Repeated hearings of good performances, in addition to study, were essential to proper familiarity and understanding, as the more enlightened critics of new music had always pointed out. Moreover, as the pianola, gramophone and radio were to show, the innovations of technology could transform the listening and learning experience by providing easy access to repetition and reinforcement. In the days when such marvels were unimaginable, programme notes could of course help the reader recall some of the music in tranquillity (Bashford 2003, p. 131).

5 Les nouveaux modes de communication touchent toutes les formations musicales : les grands ensembles symphoniques, les ensembles de musique de chambre, mais aussi les musiciens solistes qui peuvent entretenir, grâce aux réseaux sociaux, une relation suivie avec leur public respectif. Dans cet article, nous nous intéresserons en particulier au répertoire symphonique et à la manière dont les orchestres développent, à l'ère numérique, leurs outils de médiation pour ce même répertoire. 
Le format écrit, à lui seul, ne suffit pas à combler l'expérience d'apprentissage et de préparation à un concert. Aujourd'hui, Internet et ses nombreuses sources audio/ vidéo revêtent un rôle presque indispensable en la matière. Dans les années 1920, le contact avec l'œuvre passait aussi par l'audition, mais exclusivement par la radio, le gramophone et d'autres dispositifs similaires ${ }^{6}$.

Cette prise de conscience des limites de l'écrit et de la plus grande place à accorder à l'audition transparaît également dans les intentions de Donald Tovey (1875-1940). Musicologue, compositeur et chef d'orchestre, ce dernier est aussi l'auteur des notes de programme du Reid Orchestra qu'il a lui-même fondé en 1917 lorsqu'il était professeur à l'Université d'Édimbourg. Dans ses notes, Tovey innove à sa manière : il choisit de réduire la partie écrite et augmente celle consacrée à l'exposition des thèmes musicaux. Comme le précise Bashford:

Tovey produced programmes that were little more than a sequence of carefully chosen passages of music type, with a minimal connecting tissue of prose, and had them printed on soft, vellum-like paper. The suggestion was that attention should note (and could not) be divided between reading words and listening to sounds; that following notation was quite enough; and -implicitly - that silence during performance was a precondition at all times (Bashford 2003, p. 133).

La rigueur de l'analyse musicologique à l'écrit s'accompagne d'une certaine distance critique vis-à-vis de l'objet musical, au risque de ne pas prendre en considération l'expérience sonore initiale. L'approche orale - et aurale - vient combler cette lacune potentielle en mettant l'auditeur en prise directe avec l'œuvre. Dans L'analyse musicale, une pratique et son histoire, Kofi Agawu vante les mérites d'un apprentissage de la musique fondé sur la complémentarité entre l'écrit et l'oral. « Le savoir oral n'est pas une forme inférieure du savoir écrit », déclare Agawu. "Affirmer que le savoir oral manque de rigueur, c'est sous-estimer son potentiel ». L'auteur entend ici s'opposer à " cette tendance fort répandue qui maintient à bonne distance les mondes sonores mis en mouvement par le questionnement critique" (Agawu 2009, p. 130).

Il existe bien des modèles de pédagogie musicale qui ont su mettre en pratique cette articulation entre l'expression verbale et l'expression musicale. Dans les années 1950, notamment, on trouve deux exemples pionniers qui, à bien des égards, peuvent encore servir aujourd'hui de sources d'inspiration pour des projets de médiation, à l'ère d'Internet et des réseaux sociaux.

\section{DEUX EXEMPLES PIONNIERS}

De 1958 à 1972, le chef d'orchestre et pédagogue américain Leonard Bernstein conçoit et anime une série de concerts pour jeune public intitulée Young People's Concerts. Il se présente à la fois comme conférencier, interprète au piano et chef

6 Sur l'apparition du gramophone parmi les nouveaux modes de consommation de la musique et sur les notes discographiques qui accompagnent l'écoute de l'auditeur dans un cadre privé, voir Maisonneuve 2009. 
d'orchestre à l'occasion de 53 événements destinés aux enfants, dont l'âge est compris entre 8 et 18 ans environ (voir Gottlieb 2010, p. 175).

Ces épisodes ${ }^{7}$, retransmis sur la chaîne américaine CBS, offrent au chef d'orchestre la possibilité de présenter lui-même, oralement, des éléments contextuels, historiques et analytiques sur les œuvres qui seront exécutées. Le contenu de ses présentations varie : elles portent tantôt sur une ouvre, comme la Symphonie fantastique de Berlioz ou Les planètes de Gustav Holst, tantôt sur un compositeur, comme Beethoven ou Mahler, tantôt sur des notions musicales élémentaires, comme la définition d'une mélodie, d'un mode ou d'une forme sonate.

Dans une autre série pédagogique réalisée pour la télévision, intitulée Omnibus, Bernstein innove une fois de plus. On note, par exemple, un épisode consacré à la Symphonie $n^{\circ} 5$ de Beethoven, dans lequel le chef d'orchestre choisit d'aborder des sujets très spécifiques : la genèse, les différentes versions envisagées par Beethoven et, plus globalement, les doutes qui peuvent s'emparer de tout compositeur au cours de son processus créatif ${ }^{8}$. De ce point de vue, les épisodes de la série Omnibus, comme les Young People's Concerts, peuvent être assimilés à des notes de programme augmentées, notamment grâce au support audio-visuel, qui s'adressent aussi bien à l'auditeur dans la salle qu'à l'auditeur potentiel. Certes, il existe de nombreux points de convergence avec les capsules vidéo que nous nous apprêtons à recenser, mais, à l'époque où sont réalisées ces émissions, Bernstein est encore loin de pouvoir bénéficier du caractère interactif des outils numériques. De son côté, le public ne peut pas non plus profiter d'un espace d'expression bien défini, comme aujourd'hui sur les réseaux sociaux.

À la même période, en France, une initiative similaire voit le jour. Il s'agit d'une série de disques commentées, consacrée aux grands compositeurs de musique classique et produite sous l'étiquette Disques Adès. À l'instar des conférences-concerts de Bernstein, cette série s'adresse avant tout aux enfants et, pour chaque épisode, la présentation des œuvres s'opère principalement sur le mode de l'oral; on y entend les extraits musicaux qui illustrent les propos tenus ou qui servent à prouver un argument exposé à l'oral. L'utilisation du support audio et le large écoulement des disques sur le marché garantissent à cette série une grande accessibilité, comparable à celle des concerts avec médiation intégrée de Bernstein.

Les premiers enregistrements de la collection à paraître mettent à l'honneur Mozart, Chopin, Schubert, Bach, Beethoven ou encore Liszt. Sur la pochette des « 33 tours »,

$7 \quad$ Afin de constater le résultat que produisent ces concerts diffusés à la télévision, nous pouvons nous référer aux nombreux épisodes disponibles sur YouTube. Voici un exemple parmi tant d'autres : "Berlioz takes a trip ", que l'on peut traduire par " Berlioz part en voyage [psychédélique] ", disponible à l'adresse suivante : https://www.youtube.com/watch?v=tWrut6bxK0M (consulté le 17 janvier 2020).

8 Nous avons la chance d'avoir accès non seulement au script de l'épisode, mais également à l'épisode lui-même. Bernstein, alors âgé de 36 ans, y explique la genèse de l'œuvre en ayant les pieds posés sur une partition géante. Diffusé pour la première fois le 14 novembre 1954, cet épisode témoigne d'un travail méticuleux de mise en scène et de scénographie. Il s'agit là d'une des vidéos les plus fascinantes qui nous a été donnée de voir au cours de nos recherches. Disponible à l'adresse suivante : https://www.youtube. com/watch?v=lrDyvxSnqb0 (consulté le 15 janvier 2020). En ce qui concerne le texte intégral, voir Bernstein 1959, p. 73-93. 
chaque nom de compositeur est suivi de la mention " raconté aux enfants » ou de "sa vie, ses œuvres ", ce qui produit un effet de série au sein même de la collection. À la fin des années 1960, d'autres parutions s'y ajoutent, notamment sur Schumann, Berlioz, Vivaldi, Wagner, Tchaïkovski, Debussy et Mendelssohn ${ }^{9}$. Quelques livres audio sur des œuvres musicales paraissent également : L'apprenti sorcier de Dukas, Pierre et le loup de Prokofiev, Les scènes d'enfants de Robert Schumann, ainsi que l'opéra La flûte enchantée de Mozart, raconté par Claude Rich ${ }^{10}$.

Malgré les nobles intentions de Lucien Adès, éditeur des Disques Adès et créateur de la série du "Petit Ménestrel ", force est de constater que ces enregistrements ne donnent que peu de clés de compréhension sur la musique elle-même ${ }^{11}$. Bien qu'elle fourmille d'anecdotes et de citations sur la vie des compositeurs - informations qui à elles seules ne peuvent expliquer la complexité ni la structure d'une œuvre -, cette médiation demeure un outil d'éveil musical seulement, mais ne couvre pas autant de sujets qu'une note de programme ou qu'une conférence-concert telle qu'imaginée par Bernstein. De plus, contrairement aux initiatives de Bernstein et à d'autres ayant retenu notre attention, les disques de la collection "Le Petit Ménestrel " sont destinés à être écoutés pour eux-mêmes, sans lien avec un concert ou un programme de concert pour lequel des notes ont été écrites.

Aujourd'hui, la plupart des orchestres symphoniques eux-mêmes possèdent aujourd'hui leur propre chaîne YouTube et publient leur propre contenu audio ou vidéo. Nous nous attarderons maintenant sur quelques initiatives de ce type et, ultérieurement, sur un projet de capsules vidéo semblable, que nous avons nous-mêmes réalisé en partenariat avec l'Orchestre symphonique de Montréal (OSM).

\section{PROJET DE CAPSUleS VIDÉO : QUELQUES MODĖLES DÉJÀ EXISTANTS}

Comme Bernstein avant lui, le chef d'orchestre Michael Tilson Thomas a porté plusieurs grands projets de médiation. Son intérêt et son talent de pédagogue l'ont amené à se présenter devant un large public, dans le cadre des prestigieuses conférences TED. Directeur musical du San Francisco Symphony, Tilson Thomas est également le créateur et narrateur d'une série de documentaires produite par la chaîne de télévision

9 Du côté des narrateurs, certains grands noms du théâtre et du cinéma prêteront leur voix à l'exercice, comme Francis Huster dans une réédition d'un disque consacré à Liszt et publié en 2008.

10 Peu d'informations existent aujourd'hui sur les parutions de la collection « Le Petit Ménestrel ». Les catalogues disponibles sur Internet sont ceux de bibliothèques ou de revendeurs et donc inévitablement incomplets. Voir par exemple le site de la $\underline{B N F}$ ou encore celui du revendeur Discogs. Parmi les quelques exemples de la collection accessibles sur YouTube, nous pouvons citer le récit de la vie de Mozart, parsemé de plusieurs extraits de ses œuvres. Celui-ci est emblématique puisqu'il est raconté par le grand Gérard Philippe, qui a également prêté sa voix à Pierre et le loup. Disponible à l'adresse suivante : https://www.youtube.com/watch?v=FBv3HS JgzI (consulté le 15 janvier 2020).

11 Dans cet exemple de narration sur la vie de Mozart, Gérard Philippe raconte essentiellement les péripéties, les joies et les épreuves du jeune compositeur. Il évoque l'influence de Bach et de Haendel sur sa composition de la Grande messe en do mineur, mais ne précise pas en quoi elle consiste. En terminant son « entretien amical » avec ses auditeurs, le narrateur dit simplement de la Symphonie $n^{\circ} 41$ qu' « elle fait éclater la force et l'invention ». 
américaine PBS, appelés Keeping Score. Inaugurée en 2006, cette série n'a malheureusement duré que quelques années, jusqu'en $2011^{12}$.

Autre outil de médiation du San Francisco Symphony à souligner : la série Program Note Podcasts ${ }^{13}$. Celle-ci met à la disposition des internautes les notes de programme en prévision des concerts, mais avec la particularité de les présenter sous forme orale $^{14}$. L'alternance entre narration et diffusion d'extraits musicaux sans visuel est aussi l'approche que nous adopterons nous-mêmes dans les premières capsules vidéo de notre projet.

En 2014, à l'occasion du Free College Concert, évènement annuel qui donne la chance aux étudiants universitaires d'assister gratuitement à un concert de musique classique dans une ambiance décontractée, le Philadelphia Orchestra lance une nouvelle application appelée LiveNote ${ }^{15}$. Cet outil original, proprement numérique, permet aux auditeurs de suivre en temps réel l'œuvre musicale pendant qu'ils sont assis dans la salle de concerts. Grâce à une technologie déjà existante de reconnaissance d'extraits musicaux, telle qu'en ont aussi profité les développeurs Shazam et SoundHound, LiveNote accompagne le public dans son écoute, offrant une analyse approfondie de l'œuvre à travers ses thèmes et ses motifs les plus marquants ${ }^{16}$.

D'autres orchestres, comme le Philharmonia Orchestra et 1'Orchestre national d'Île-de-France, ont grandement œuvré et continuent d'œuvrer dans le domaine de la médiation musicale. L'orchestre londonien, sous la direction de Esa-Pekka Salonen, héberge notamment plusieurs guides d'écoute ("Listening guides ») sur son site Internet officiel, sous l'onglet « Films ${ }^{17}$.

Le format de ces guides varie, tantôt avec un spécialiste du sujet, au ton austère et en situation d'entrevue, tantôt avec un acteur qui livre le texte de manière plus enjouée ; tantôt avec le minimum d'effets visuels et de montage, tantôt avec un florilège d'animations. Parmi les vidéos disponibles, on trouve une variété d'œuvres connues et méconnues que l'orchestre cherche à démystifier pour le grand public : des symphonies de Beethoven et de Mahler, la Symphonie fantastique de Berlioz, le style de Stravinski (Le sacre du printemps, L'oiseau de feu), mais aussi des œuvres de

12 Pour la liste de complète des documentaires et des différents outils de médiation offerts par Keeping Score, voir le site Internet de la série.

13 Voir la page spécialement consacrée à cette initiative.

14 Sur cette page web, on peut entendre un enregistrement audio, parmi d'autres, centré sur la vie de Brahms et son identité musicale à travers ses symphonies et des éléments d'analyse musicale, notamment sur la Symphonie $n^{\circ} 1$ au programme de la saison (les dates affichées sont les $1^{\text {er }}$ et 3 mars 2018). On y entend la voix du narrateur Rick Mallone pour une durée totale de 13 minutes et 49 secondes. À noter que le contenu n'est qu'audio ; l'apport de la vidéo aurait permis de montrer, par exemple, des extraits de partition pendant la partie de l'enregistrement consacrée à l'analyse musicale.

15 Voir le site officiel du Philadelphia Orchestra. Voir aussi l'article de présentation de l'application, publié le 2 octobre 2014, sur la page suivante : https://www.philorch.org/press-room/news/philadelphia-orchestra-launches-livenote ${ }^{\mathrm{TM}}$-interactive-concert-guide-mobile-devices\#/ (consulté 15 janvier 2020).

16 À noter que l'utilisation de l'application est aujourd'hui encadrée ; elle n'intervient que lors des concerts de la série LiveNote Nights, moments durant lesquels l'utilisation des appareils mobiles est autorisée dans l'enceinte du Verizon Hall.

17 Voir la page spécialement dédiée du Philharmonia Orchestra. 
Vaughan Williams, de Copland et de Witold Lutosławski. Les idées de mise en scène semblent par moments mal adaptées au propos sérieux et bien documenté, mais elles témoignent d'un vrai effort de médiation musicale. Chaque capsule ou presque contient des extraits de partition et certains thèmes musicaux mis en exergue par des effets visuels ; une technique que nous emploierons plus tard dans la conception graphique de nos propres vidéos.

De son côté, l'Orchestre national d'Île-de-France (ONDIF) a inauguré une série de vidéos sur son site Internet, directement reliée à sa chaîne YouTube et intitulée Allez, raconte Camille. Le prénom renvoie à celui de la responsable du contenu et de la présentation, Camille Villanove, accompagnée par Bernard Chapelle en tant que coanimateur (à la réalisation, Laurent Sarazin). Cette série constitue l'une des expressions les plus abouties de vulgarisation musicale, au sens positif et parfois péjoratif que l'on entend : présentation condensée en moins de trois minutes, simplification et raccourcis jugés nécessaires, propos volontairement ludique pour décontracter l'ambiance autour d'un répertoire souvent perçu comme austère, nombreuses références à des objets de la culture populaire pour rejoindre le plus grand nombre. Les deux intervenants se répartissent les rôles : Camille dans celui de l'initiée et Bernard dans celui du novice, faussement naï. Cette stratégie a le double avantage de permettre à la fois au connaisseur et au néophyte de s'identifier à l'un ou l'autre des personnages, sans qu'aucun complexe de supériorité ou d'infériorité ne soit véhiculé ${ }^{18}$.

À l'instar de l'initiative du Philharmonia Orchestra, celle de l'onDIF offre au jeune public et au public adulte des outils de médiation adaptés. C'est aussi le sens de notre projet de capsules vidéo, réalisé en partenariat avec l'osm. Nous avons notamment cherché à stimuler des réactions positives du public, par un design graphique et des références culturelles plus proches de l'auditeur d'aujourd'hui.

Il va sans dire que le processus créatif en amont de chaque nouvelle capsule vidéo à réaliser s'est nourri de réflexions et d'interrogations sur la manière d'améliorer constamment ces outils de médiation numériques. Plusieurs questions récurrentes se sont posées à nous : comment tirer au mieux parti des technologies et logiciels actuels pour concevoir des notes de programme incorporant de l'audio-vidéo ? Quels sont les atouts et les limites de celles-ci par rapport au format écrit? Quels sont leurs objectifs et leurs approches musicologiques ? Quelles méthodes de réalisation pouvons-nous établir de manière plus systématique afin de gagner en efficacité ? Enfin, comment les capsules vidéo sont-elles reçues ou bien perçues par les internautes ? Ce sont toutes ces questions auxquelles nous tenterons maintenant de répondre.

18 Voir la liste des vidéos disponibles sur Dailymotion - équivalent français de YouTube - pour la série Allez, raconte Camillle. À noter qu'à partir du mois d'octobre 2017, l'ondif a mis en ligne une nouvelle web-série, intitulée MarmitOndif, avec la musicologue Corinne Schneider en remplacement de Camille Villanove. Déjà présents lors de la précédente série, Bernard Lachapelle, à l'animation, et Laurent Sarazin, à la réalisation, poursuivent ici leur collaboration avec l'orchestre francilien. Voir cette autre liste de vidéos sur YouTube. 


\section{DESCRIPTION DU PROJET DE CAPSULES VIDÉO (DPMQ-OSM)}

Le projet, intitulé «notes de programme numériques », a été réalisé sous l'impulsion commune de l'Observatoire interdisciplinaire de création et de recherche en musique (OICRM), par le biais de l'équipe de recherche sur le Développement des publics de la musique au Québec (DPMQ ${ }^{19}$ ), et de l'OsM. Il a permis de jeter les bases d'une riche collaboration entre le milieu de la recherche et le milieu professionnel, notamment à travers six capsules vidéo en ligne, consacrées à une ou plusieurs œuvres au programme de la saison 2015-2016 ${ }^{20}$. Réalisé dans le cadre d'un stage au sein de l'administration de l'osm, ce projet s'est échelonné sur une période de quatre mois, débutant officiellement le 15 juin et prenant fin le 15 octobre 2015 (période couverte par une subvention de l'organisme Mitacs).

Parmi les employés et responsables que nous avons été amenés à côtoyer figurent, par ordre hiérarchique, Jean-Claude Bernier, rédacteur et coordonnateur des programmes et, en l'occurrence, notre superviseur direct ; Véronique Boileau, directrice des Relations publiques ; Marie-Josée Desrochers, en charge de la planification et du rayonnement international, devenue chef de l'exploitation à la faveur d'une restructuration au sein de l'entreprise.

Le stage nous a donné l'occasion de voir de près l'organisation du travail à l'osm et tous les mécanismes en jeu lors de la préparation de matériels destinés à la médiation, plus particulièrement celle des notes de programme ${ }^{21}$. Il nous a également permis de développer une habileté spécifique à la rédaction de notes de programme numériques. Les trois premières capsules vidéo, auxquelles s'ajoutent trois autres réalisées entre le 15 octobre 2015 et le 30 avril 2016, témoignent de l'amélioration de nos compétences en la matière. De plus, nous avons su mieux anticiper les attentes de nos superviseurs à l'osm. Il ne s'agissait pas, en effet, de réaliser un travail dans un cadre universitaire, mais bien pour une entreprise partenaire. Les capsules vidéo devaient être adaptées à un format d'au maximum 3 minutes 30 secondes et ainsi contenir l'essentiel des connaissances musicologiques au sujet de l'œuvre présentée.

\section{CONSTITUTION DE DIFFÉRENTS MODÈLES}

Nous avions pour responsabilité de rédiger une série de notes de programme, indépendantes de celles publiées dans les programmes de concert et spécialement conçues pour être mises en musique et en image. Sous forme de narration, dans un premier

19 Le DPMQ a depuis changé de nom. Il se nomme aujourd'hui Partenariat sur les publics de la musique. Voir son nouveau site Internet.

20 Trois capsules vidéo réalisées pendant la durée officielle du stage (mi-juin à mi-octobre 2015) et trois autres qui se sont échelonnées jusqu'à la fin de la saison (mi-octobre 2015 à fin-avril 2016). Nous reviendrons plus tard sur le déroulement de ces deux périodes.

21 Outre les programmes de concert de la saison en cours, qui étaient le fruit du travail quotidien de Jean-Claude Bernier, nous avons pu consulter la banque de notes de programme disponible aux archives de l'osm et qui remonte à la création de l'organisme, en 1934. Cette ressource est venue compléter les recherches que nous avions entamées sur les œuvres faisant l'objet de capsules vidéo. 
temps, le contenu musicologique était accompagné par un ensemble d'animations graphiques, captures visuelles de partitions défilantes, reproductions d'images, portraits de compositeurs et, bien sûr, extraits sonores de l'œuvre au programme. Avec l'aide de Jason Ghikadis, designer graphiste ayant des compétences en musique et collaborateur du DPMQ, nous avons pu travailler dans les meilleures conditions à la réalisation de ces capsules vidéo. Au terme du stage officiel couvert par notre subvention, d'autres capsules ont été réalisées, mais cette fois-ci sur un second modèle, sans narration. En l'absence de voix-off, l'expression écrite a été privilégiée : des phrases reproduites en surimpression, le plus souvent comme sous-titres, apparaissaient au-dessus des images en mouvement. Entre les deux modèles - avec narration ou avec sous-titres -, les méthodes de travail demeuraient essentiellement les mêmes.

La direction de l'osm tenait à ce qu'il y ait une introduction accrocheuse (hook) qui puise attirer d'emblée l'attention de ceux qui visionneraient la capsule sur Internet. Une partie des choix musicologiques ont été faits en fonction de cette exigence : nous avons multiplié les allusions à la culture populaire et, avec l'aide du graphiste, nous nous sommes inspirés de référents visuels facilement identifiables pour un public de tous âges. Compte tenu du format condensé des capsules, nous avons dû faire une sélection rigoureuse des nombreux éléments d'information disponibles sur l'œuvre musicale et son compositeur.

À ces difficultés s'ajoutaient des considérations plus pratiques. Pour être conforme à la loi, il fallait trouver des contenus libres de droits ou débourser un certain montant pour pouvoir les exploiter. Ainsi, nous avons sélectionné une quantité d'images faisant désormais partie du domaine public. Les images protégées par le droit d'auteur, quant à elles, ne devaient pas être reproduites à l'identique, mais modifiées ou détournées.

L'obtention des droits d'utilisation d'extraits audio était de la responsabilité du service Marketing. Une entente avait déjà été signée avec la compagnie de disques Naxos et, contre un montant supplémentaire, l'équipe administrative a pu bénéficier d'une diffusion plus large d'extraits sur les réseaux sociaux (plateformes Facebook, Twitter et YouTube). Le projet de capsules vidéo s'inscrivait dans ce cadre légal, nous permettant d'établir un contact régulier avec les membres du service Marketing.

$\mathrm{Au}$ cours du stage de quatre mois, nous avons pu nous appuyer sur la coopération de nombreux acteurs à l'osm ${ }^{22}$. Entourés de nos superviseurs, dans un espace de travail ouvert (open-space), nous avons profité de cette proximité pour les tenir informés, jour après jour, du déroulement de chaque projet. Nous avons également travaillé étroitement avec les équipes des Relations publiques, des Communications et du Marketing ainsi que de l'Éducation. Nous nous trouvions en immersion totale au sein de l'entreprise, ce qui témoigne de la singularité du partenariat entre le DPMQ et l'OSM.

La production des différentes vidéos a donné lieu à de nombreux allers-et-venues entre nos superviseurs et nous-mêmes. Elle se déroulait principalement en

22 Comme nous le verrons plus tard, à partir du 15 octobre 2015 et la fin officielle de notre stage à l'osm, le nombre de personnes impliquées dans la production des capsules vidéo sera réduit, notamment à cause d'une restructuration à l'interne. 
neuf étapes : 1) recherches et rédaction d'une note de programme préliminaire, 2) approbation du texte par nos superviseurs ${ }^{23}, 3$ ) élaboration d'un storyboard ${ }^{24}$ afin de mettre ce texte en images ${ }^{25}, 4$ ) approbation du storyboard, 5) choix du narrateur et enregistrement de la narration à partir du texte préliminaire, 6) incorporation de la narration sur les animations visuelles et création d'une première version de capsule vidéo, 7) approbation de nos superviseurs, 8) remise d'une ultime version en haute résolution et, enfin, 9) diffusion de la capsule sur les réseaux sociaux.

Le modèle avec narration a été le premier à être mis en place. Plusieurs versions de notes ont été rédigées ; des textes préparatoires ont ensuite servi de base pour la narration ${ }^{26}$. Il était donc essentiel d'envisager le style et les formules d'écriture dans la perspective $\mathrm{du}$ discours oral. Le contenu musicologique se traduisait également en images par des reproductions et des versions simplifiées de la partition, dont certains passages étaient mis en exergue par des cercles de couleurs. En comparaison, le modèle avec sous-titres ne nécessitait pas autant d'intervenants pour la préparation d'une capsule vidéo.

La question est alors de savoir lequel des deux modèles est le plus adapté pour le public de concerts, jeune ou plus âgé, novice ou plus expérimenté. La narration permet d'écouter le contenu en toute simplicité, au risque d'adopter une attitude plus passive quant à l'exercice de compréhension. Les sous-titres offrent plus de matière pour assouvir la curiosité du lecteur, mais ils requièrent aussi une lecture plus ou moins rapide du texte défilant et donc une attention accrue pour saisir pleinement le contenu des capsules. Tandis que la narration accumule inévitablement du son par-dessus la musique - et est donc susceptible d'entrer en conflit avec les extraits de l'œuvre qui méritent d'être écoutés attentivement -, les sous-titres permettent à la musique de s'exprimer pleinement ${ }^{27}$. En revanche, ces derniers peuvent entrer potentiellement en conflit avec l'espace de l'écran destiné à l'animation visuelle. Il revient au graphiste d'organiser adéquatement l'espace de l'écran disponible ; il revient aussi au monteur de gérer l'équilibre sonore entre narration et musique. La conception de capsules impose alors de coordonner et d'articuler ces trois

23 Chaque étape d'approbation impliquait souvent plusieurs allers-et-venues entre les superviseurs, le réalisateur et le graphiste de la capsule : en résultaient des commentaires et demandes de correction à prendre en compte dans les versions ultérieures à l'original.

24 Nous choisissons le terme en anglais car, à notre connaissance, il est plus communément employé que son équivalent en français « scénarimage».

25 Sous forme de feuille de route (cue list), ce document consignait toutes les illustrations et les extraits sonores qui devaient accompagner le fil de la narration. Voir, en annexe, une copie de storyboard, à titre d'exemple.

26 Tous les enregistrements de narrations et les montages ont été effectués aux studios de Radio vM (anciennement Radio Ville-Marie). De par notre travail bénévole en tant qu'animateur et réalisateur à la station de radio, nous avons pu bénéficier en contrepartie d'un service professionnel gratuit, dans un lieu tout destiné. Ce service est venu d'un technicien qui faisait déjà la prise de son et le montage de nos propres émissions radiophoniques.

27 Dans ces capsules vidéo, le rôle de la musique n'est pas exclusivement dévolu à l'accompagnement sonore et expressif des images, mais surtout à la justification du contenu musicologique. Qui plus est, la musique est elle-même porteuse de significations essentielles rattachées à l'œuvre. Citant les théories d'Aurélie Igonet sur l'architecture et la structuration du son tel que compris par l'auditeur, Béchemin, Cohen et Rampnoux ont cette formule : "le statut du son se transforme de producteur d'émotion en producteur de sens ». Pour plus de détails, voir Igonet 2005, p. 21. 
formes syntaxiques (visuelle, textuelle et sonore) sans que celles-ci se fassent concurrence de manière frontale et évidente pour le spectateur-auditeur. La qualité d'une capsule se mesurera essentiellement par la maîtrise de tous les éléments de syntaxe et par l'harmonie qui s'en dégage.

Compte tenu du fait que le son des vidéos sur Facebook est coupé par défaut - il faut en effet cliquer sur la vidéo ou augmenter le volume sur la vidéo pour actionner le son -, le modèle avec sous-titres peut s'avérer utile. Nous croyons, néanmoins, que le contenu musicologique bénéficie d'un meilleur traitement grâce à la narration. Par l'intermédiaire de la voix, la capsule s'adresse plus directement à l'auditeur. Elle lui transmet, de manière plus fluide, des connaissances sur l'œuvre et le compositeur. Certains éléments textuels peuvent aussi être ajoutés pour renforcer une idée énoncée à l'oral, ce qui vient compléter tout l'éventail de possibilités d'une capsule avec narration.

\section{CHOIX DE LIGNE ÉDITORIALE}

Au total, cinq concerts ou événements ont fait l'objet de capsules vidéo. Il convient de rappeler les trois premiers : le concert de clôture de la Virée classique, édition 2015 (Les pins de Rome de Respighi), le concert d'ouverture de la saison 2015-2016 (Pelléas et Mélisande de Debussy) et la série de concerts sur la thématique d'Halloween. Deux capsules vidéo ont été programmées pour le festival Tchaïkovski, l'une sur le compositeur et l'autre sur une de ses œuvres majeures : la Symphonie $n^{\circ} 6$. Enfin, une série de deux concerts, présentée les 11 et 12 mai 2016, a donné lieu à une dernière capsule sur la Symphonie $n^{\circ} 1$ de Mahler.

Le choix des œuvres a été celui de Marie-Josée Desrochers qui, pour ainsi dire, nous commandait les capsules vidéo. Ce choix a été guidé par une volonté de cibler différentes œuvres dans différents contextes : deux festivals, deux concerts de musique symphonique, un concert d'ouverture et un concert thématique avec six courtes œuvres au programme. Certains de ces événements, comme les trois premiers, étaient jugés " en difficulté ", d'après les chiffres communiqués par le service des ventes et de la billetterie ${ }^{28}$. Outre les campagnes publicitaires menées quelques semaines avant le concert de clôture de la Virée classique, avant le concert d'Halloween et, dans une moindre mesure, dans les semaines précédant le concert d'ouverture de la saison, les capsules venaient alimenter les messages promotionnels en vue des concerts, notamment sur les réseaux sociaux - nous reviendrons plus en détail sur la manière dont s'est déroulée la préparation de chacune des capsules vidéo. La direction de l'osm optait donc pour une multiplication, voire une confusion des rôles qu'elle souhaitait faire jouer à ces vidéos : outils de développement de public, outils de médiation, à la manière d'une note de programme traditionnel et outils de promotion ${ }^{29}$.

28 Ces chiffres demeurent confidentiels. Nous ne faisons que rendre compte de certaines conversations entre nos superviseurs et nous-mêmes.

29 Le fait que nous étions rattachés aux Relations publiques et entretenions plus de contacts avec le service Communications et Marketing qu'avec celui de l'Éducation accrédite plutôt la thèse d'un rôle promotionnel. Or, dans les faits, le travail réalisé sur ces vidéos n'était pas orienté vers cet objectif. 
Au mois de février 2015, soit quatre mois avant le début de notre stage, nous avions réalisé une capsule prototypique qui avait déjà permis aux responsables de l'osM d'évaluer les points forts à consolider et les points faibles à corriger. C'est par cette première réalisation que nous débuterons notre exposé.

\section{L'ouverture de Guillaume Tell de Rossini}

En amont de notre projet, nous avons travaillé sur un prototype consacré à l'ouverture de Guillaume Tell de Rossini. Le produit final a été livré à l'osm et publié sur Facebook le jour même du concert où était présentée cette pièce, le 19 février 2015. Puisqu'il ne s'agissait encore que d'un projet pilote, Jason et moi ${ }^{30}$ avons eu, à cette époque, une liberté totale pour concevoir notre toute première capsule, ce qui représente un fait exceptionnel ${ }^{31}$. Nous avons, par exemple, ajouté un court clip vidéo du générique d'ouverture de The Lone Ranger, série télévisée américaine des années 1950 qui utilisait la musique de Rossini comme bande sonore. De plus, nous avons reproduit plusieurs images de paysages suisses, notamment à travers des tableaux du peintre Ferdinand Hodler, en référence à ce personnage mythique et figure symbolique de l'indépendance suisse.

Extrait vidéo 1: Capsule vidéo sur l'ouverture de Guillaume Tell de Rossini. Voir la vidéo.

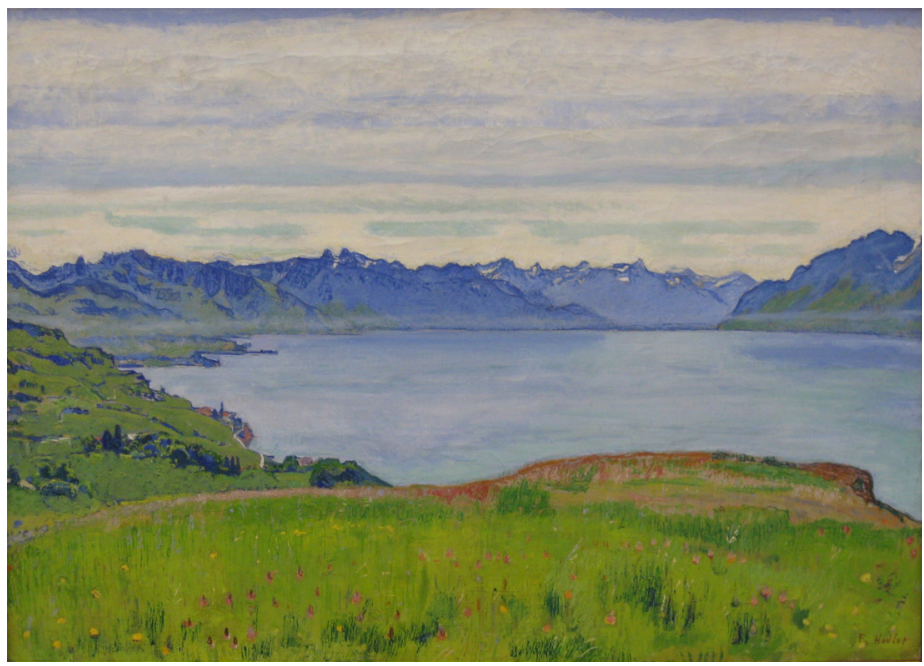

Figure 1 : Vue sur le lac de Genève (1906) de Ferdinand Hodler.

En partie à cause de ces reproductions et des portraits d'époque, les responsables des services concernés de l'osm ont jugé, rétrospectivement, que la facture de cette

30 Pour nous assurer de la compréhension du lecteur, nous optons pour la première personne du singulier. Ainsi, nous pourrons distinguer notre travail de celui de Jason Ghikadis, le « nous » englobant à la fois le réalisateur et le graphiste. Auparavant, une telle différentiation n'avait pas lieu d'être, car nous étions seuls à préparer le projet en amont.

31 Comme nous l'avons expliqué précédemment, les projets subséquents allaient en effet nous contraindre aux exigences du service Communications et Marketing de l'osm. 
capsule n'était pas assez dans l'ère de notre temps ${ }^{32}$. Pourtant, Jason et moi avions déjà jeté les bases d'une animation visuelle de la partition avec des éléments d'analyse musicale, mouvement par mouvement, qui allait devenir une marque de référence pour les projets de capsules futurs. L'organisation du contenu, la présentation et le rythme de l'information communiquée par la narration se sont approchés de l'idéal que nous cherchions à atteindre, plus que beaucoup d'autres projets ayant été brusqués par le temps et les nombreuses révisions.

Les pins de Rome de Respighi

Les qualités d'orchestration de l'œuvre, notamment du quatrième mouvement, m'ont rapidement orienté vers le choix de faire plusieurs références aux musiques de film, comme à celles de John Williams dans La guerre des étoiles et Superman.

Les mouvements de ce poème symphonique racontent, chacun à leur manière, différentes scènes de jour et de nuit à l'ombre des grands pins, arbres symboliques de Rome. J'ai donc rédigé un texte qui rendait compte de ces péripéties, d'un mouvement à l'autre. Pour illustrer le propos, j'ai sélectionné plusieurs extraits musicaux de l'œuvre qui imitent l'ambiance des quatre lieux romains représentés : les jardins de la villa Borghese, les catacombes, la colline du Janicule et la voie Appienne.

Extrait vidéo 2: Capsule vidéo sur Les pins de Rome de Respighi. Voir la vidéo.

Comme son nom l'indique, l'œuvre rend hommage à cette végétation typique de la Ville éternelle. Le graphiste et moi avons choisi, sur Internet, plusieurs photos de pins, prises pour la plupart par des amateurs. Pour des raisons légales que nous avons déjà évoquées, celles-ci ne pouvaient être incorporées telles quelles dans la capsule. Nous les avons donc détournées par différents moyens : obstruction de certains éléments, jeux sur les filtres de couleurs et les flous. Nous nous sommes également inspirés de reproductions de tableaux, notamment ceux de William Turner (1775-1851) sur des paysages romains. En voici un exemple :

32 Quelques mois plus tard, 1'osm fait appel à des graphistes et monteurs pour sa nouvelle vidéo promotionnelle, en prévision de la saison 2015-2016. Le message à véhiculer est le suivant : l'osm présente une saison " haute en couleurs ». Pour exprimer ce message, les graphistes ont l'idée de créer l'illusion d'éclaboussures (splashs) de peinture, à la manière des drippings de Jackson Pollock (à noter toutefois que la texture des couleurs utilisée se rapproche davantage de l'aquarelle que de l'acrylique). Ainsi, en nous faisant des commentaires sur l'esthétique de notre prototype, qu'elle juge « datée », la direction de l'osm peut s'appuyer sur ses propres visuels qui, selon elle, renvoient plutôt à l'art contemporain. Voir la vidéo promotionnelle de la saison 2015-2016 : https://www.youtube.com/watch?v=70nz77-QK34 (consulté le 6 janvier 2020). 


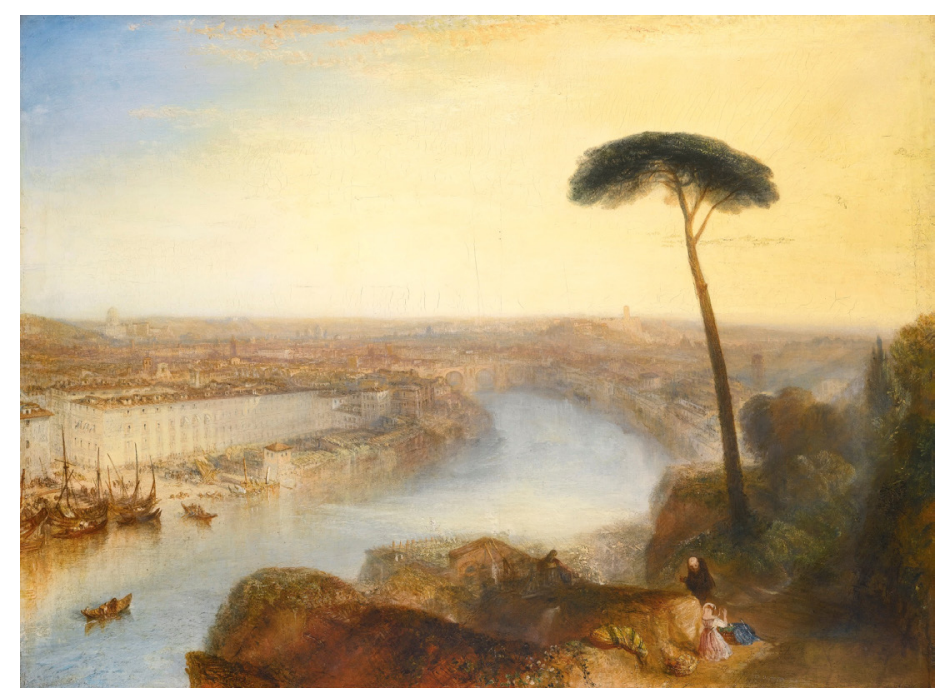

Figure 2 : Rome, From Mount Aventine (1885) de William Turner.

Lors d'une première rencontre, le graphiste, spécialisé en animations, et moi nous étions inspirés de la vidéo promotionnelle de l'osm pour la saison 2015-2016, contenant des animations de jets d'encre qui se diluent dans l'eau ${ }^{33}$; les formes ainsi créées rappelaient celles de pins, arbres au tronc élevé et au feuillage étalé. Cette animation à l'encre noire a été reprise subtilement dans la version finale. En outre, nous avons envisagé ensemble une esthétique futuriste, celle-ci appartenant à un courant artistique - le futurisme - qui est contemporain à la création de l'œuvre de Respighi, en 1924. Cette esthétique graphique ne s'est retrouvée qu'en un seul passage de la capsule, sous la forme de lignes tranchantes et dynamiques.

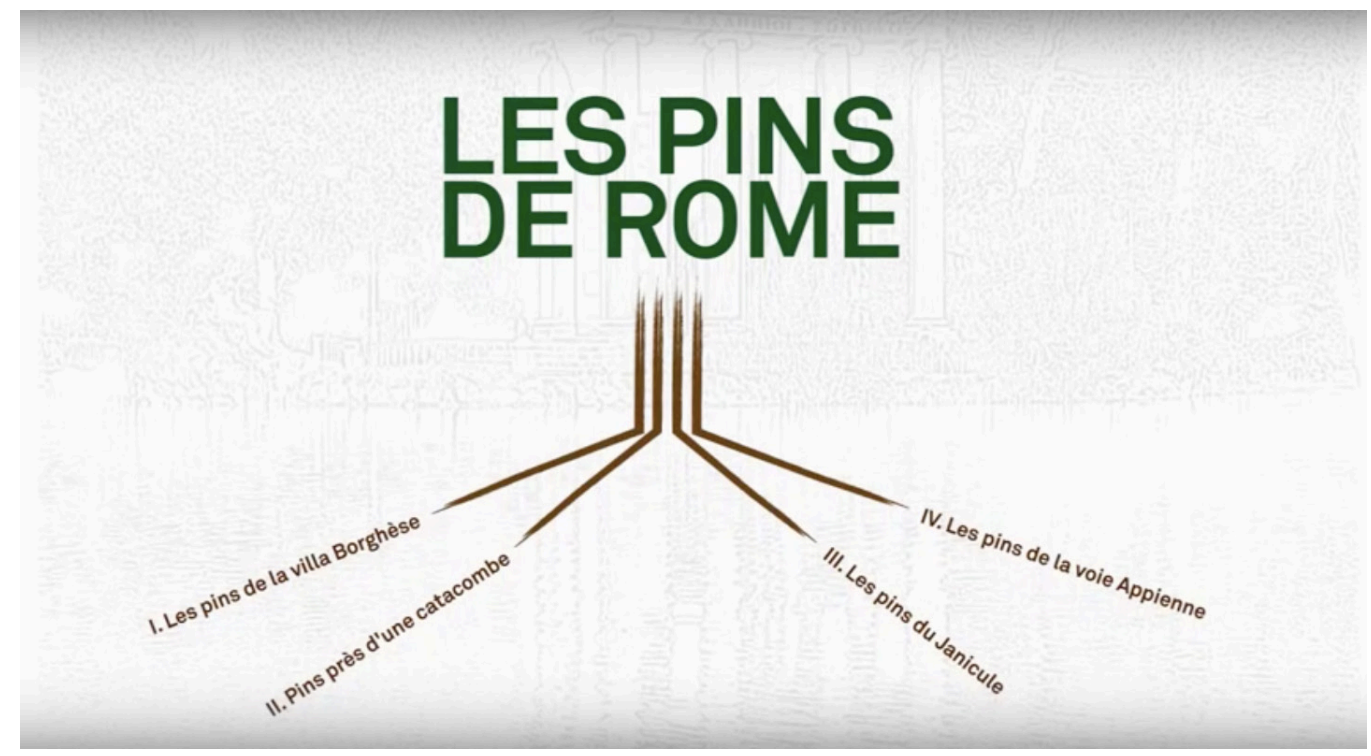

Figure 3 : Capture d'écran de la capsule vidéo sur Les pins de Rome ; esthétique futuriste (00:50). 
En raison des contraintes de temps, certains éléments d'informations ont été mis en lumière aux dépens d'autres éléments tout aussi pertinents. L'une des versions antérieures de la capsule, plus longue, mettait davantage l'accent sur le contexte politique troublé (le fascisme, Mussolini, la montée du nazisme) dans le but de susciter des discussions sur les réseaux sociaux ; une version finalement abandonnée, jugée trop polémique par l'administration de $1^{\prime} \mathrm{OSM}^{34}$.

\section{Pelléas et Mélisande de Debussy}

L'un des défis de ce projet était de condenser une œuvre aussi vaste (deux heures et demi de musique) dans une capsule de 3 minutes 30. Il fallait attirer l'attention des auditeurs par une esthétique visuelle attrayante, tout en les initiant à l'univers sonore et artistique de Debussy.

Dans une version antérieure, le texte de narration débutait par le style musical de Debussy. Il avait été coupé d'un passage portant surle conflit entre Maurice Maeterlinck, auteur de la pièce de théâtre originale, et le compositeur. C'est finalement cette partie du contexte de création, où se mêlent histoires de cœur et désirs de vengeance, qui s'est retrouvé à l'avant-plan, selon le souhait de la direction de l'osm ${ }^{35}$. J'ai imaginé, avec le graphiste, une autre référence culturelle populaire : une affiche d'un combat de boxe ou un tableau digne des jeux de combat sur console ou sur ordinateur.

Extrait vidéo 3 : Capsule vidéo sur Pelléas et Mélisande de Debussy. Voir la vidéo.

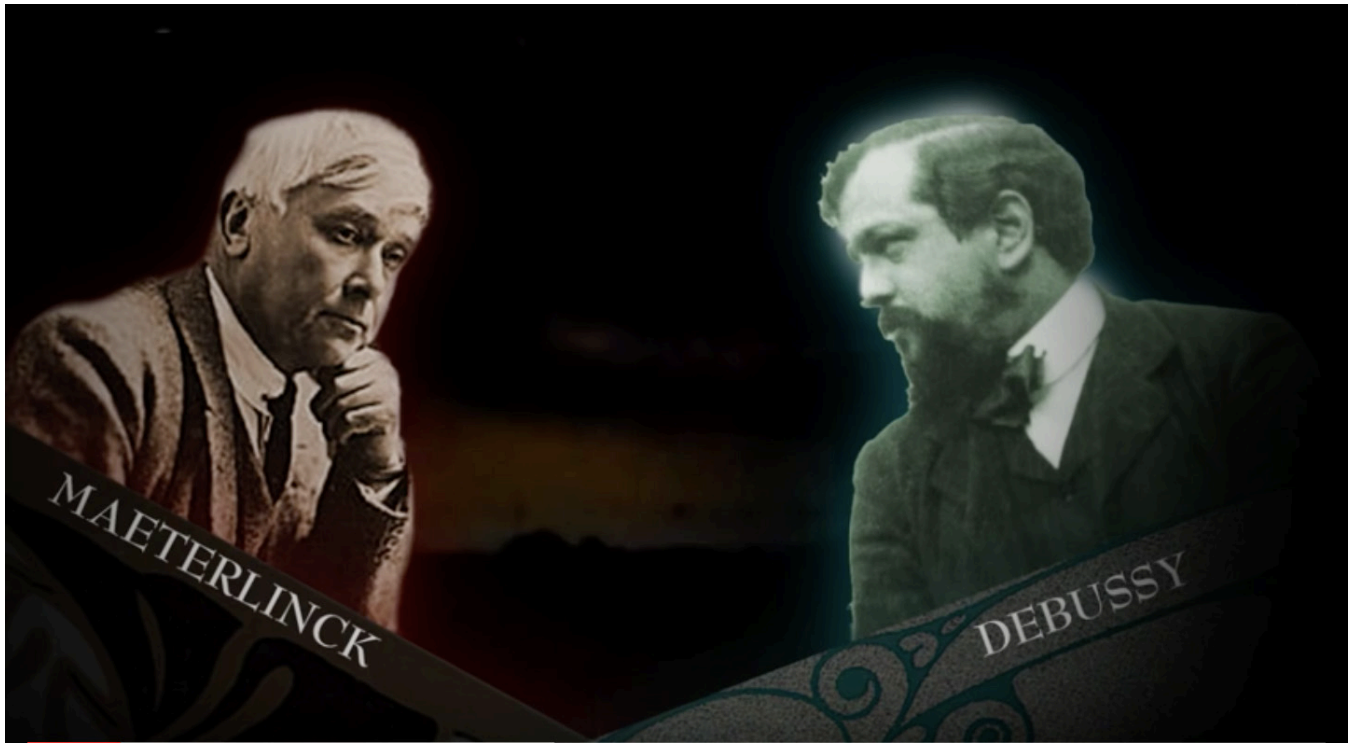

Figure 4 : Capture d'écran de la capsule vidéo sur Pelléas et Mélisande ; combat de boxe (00:16).

34 À noter une difficulté d'ordre technique, mais ayant aussi des incidences sur le choix de l'audio : le contrat de l'osm avec Naxos Canada n'était effectif qu'à partir de la saison 2015-2016 de l'organisme et non à partir de l'été 2015, correspondant à la période du festival La Virée classique. À défaut de pouvoir utiliser des extraits disponibles dans le catalogue de cette maison de disques, nous avons trouvé un enregistrement datant de 1962, paru chez RCA Records et qui faisait désormais partie du domaine public.

35 Voir, en annexe, une version antérieure du texte de narration pour Pelléas et Mélisande, où l'ordre des paragraphes est inversé. 
La correspondance et les écrits de Debussy sur Pelléas étant nombreux ${ }^{36}$, j'ai pensé incorporer quelques citations à l'intérieur de la narration. Un temps envisagée, l'idée d'une seconde voix pour réciter les paroles du compositeur a été finalement écartée. Les citations ont été uniquement retranscrites sur écran, ce qui permettait à l'auditeur de lire le passage souhaité tout en entendant distinctement la musique de fond.

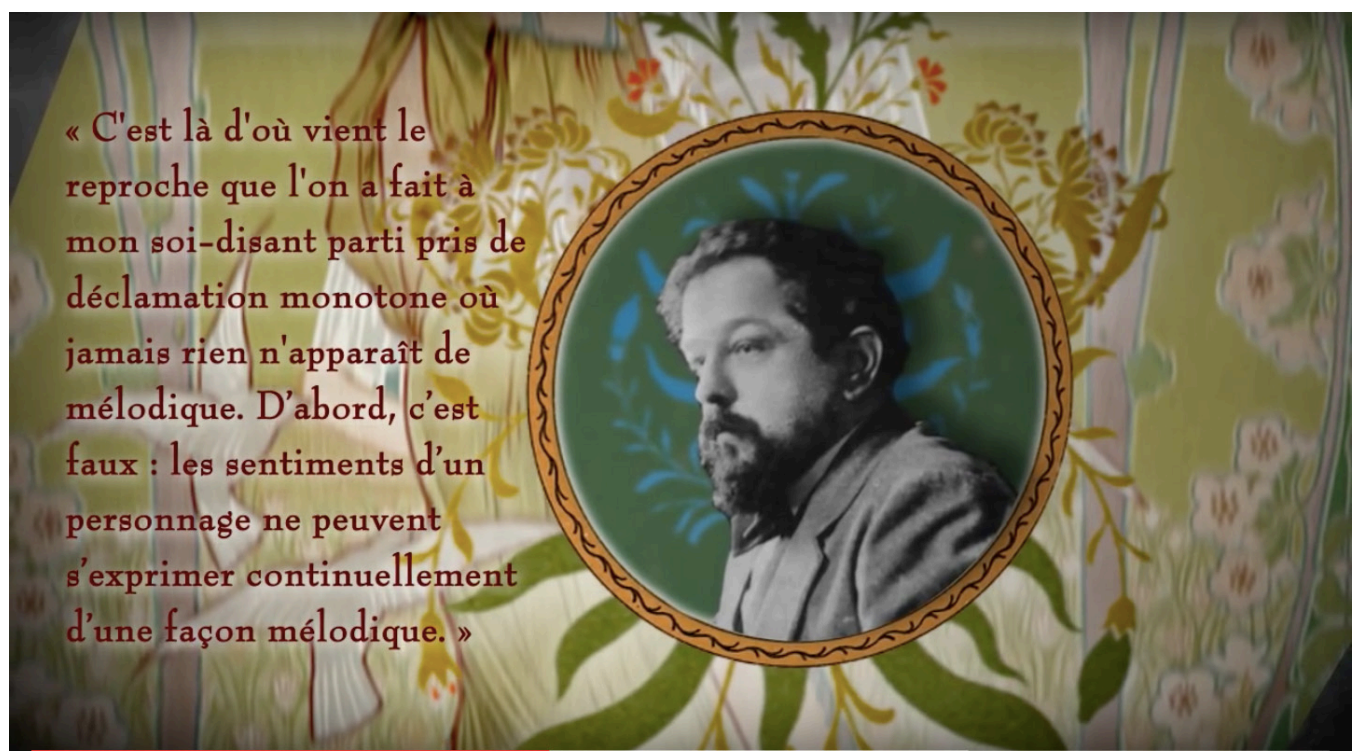

Figure 5 : Deuxième capture d'écran de la capsule vidéo sur Pelléas et Mélisande ; portrait de Debussy (01:34).

En termes d'illustrations et d'animation, le graphiste et moi avons pensé multiplier les allusions au symbolisme et à l'art nouveau, deux courants artistiques qui figuraient parmi les sources d'inspiration du compositeur. Nous voulions ainsi créer une atmosphère visuelle où, par exemple, les contours d'une arabesque se dessinent à l'écran, dans l'esprit de la mélodie debussyste.

\section{Les ceuvres du concert " Halloween "}

Contrairement à la précédente capsule, l'ordre des paragraphes m'apparaissait comme une évidence : il nous était dicté par les six œuvres au programme. Nous pouvions d'emblée attirer l'attention de l'auditeur par la thématique populaire d'Halloween, notamment grâce à une série de symboles associés à la fête des morts : la nuit, les démons, les squelettes, les sorcières, etc. Avec autant d'œuvres à couvrir, j'ai aussi pensé que nous devrions faire cinq ou six sections autonomes. Ainsi, l'osm pourrait réutiliser un fragment de la capsule à l'occasion d'un concert futur qui mettrait à nouveau l'une de ces ouvres au programme ${ }^{37}$.

36 Citons, par exemple, l'article de Debussy «Pourquoij'ai écrit Pelléas» (avril 1902), dans Debussy 1987, p. 62-64.

37 À noter que le fait de réutiliser un fragment de la capsule n'exclurait pas quelques ajustements pour tenir compte du format de capsule plus réduit (30 secondes environ). 
Mes recherches d'illustrations pour Tamara de Balakirev m'ont mené vers une toile d'Ivan Aïvazovski (1817-1900) représentant les gorges du Darial. Afin d'illustrer le mythe de cette princesse diabolique, le graphiste a reproduit ce tableau en y ajoutant quelques silhouettes de personnages évoquant l'intrigue de ce poème symphonique ${ }^{38}$.

Extrait vidéo 4: Capsule vidéo sur les auvres du concert "Halloween". Voir la vidéo.

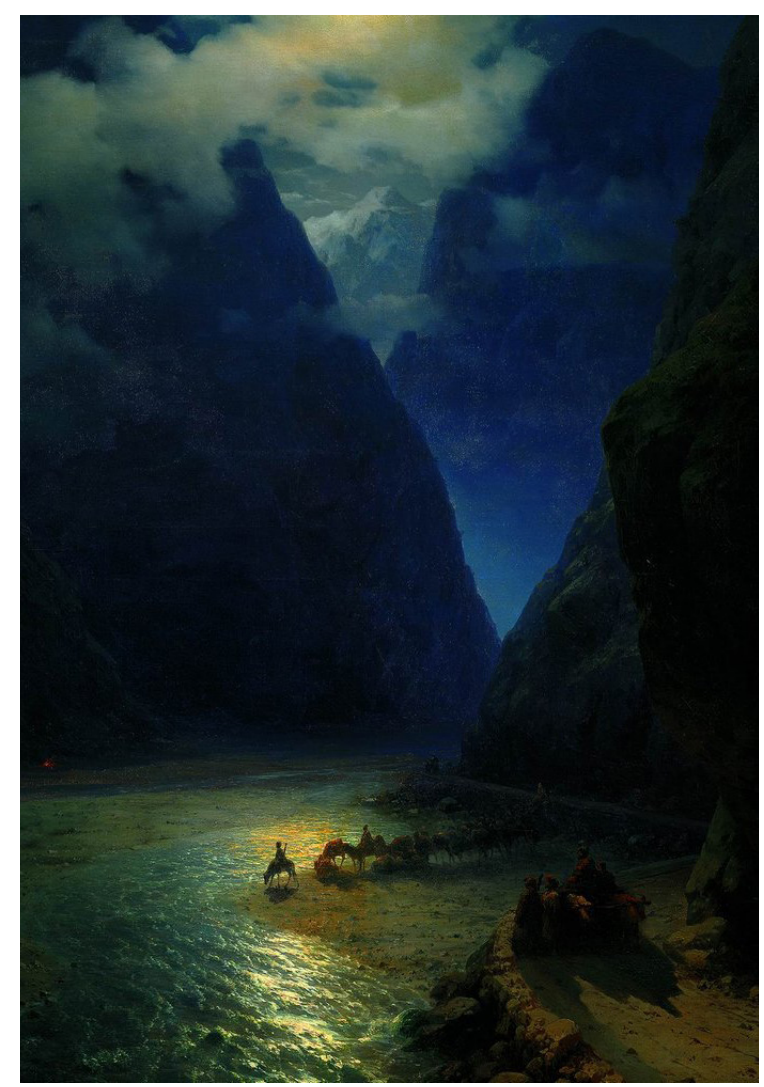

Figure 6 : Les gorges du Darial (1862) d'Ivan Aivazovski.

En ce qui concerne L'apprenti sorcier de Dukas, il était exclu de payer des droits pour l'utilisation de la célèbre séquence du film Fantasia (1940) des studios Disney, consacrée à l'œuvre du compositeur français. Néanmoins, le graphiste a pu concevoir une animation mettant en scène la marche des balais. En outre, nous avons ensemble exploré la possibilité d'utiliser le feu et le brasier comme éléments d'animation récurrents - des images censées susciter la terreur. Jason a eu également l'idée de faire une allusion au cinéma d'horreur.

Les responsables à l'osm étaient soucieux des transitions entre toutes les différentes sections de la capsule. Certains ajustements ont dû être faits en ce sens. Nous avons notamment généralisé notre utilisation d'une texture d'image qui imite la peinture à l'huile, telle qu'elle transparaît dans le tableau d'Aïvazovski. Par souci de cohérence, nous avons choisi d'illustrer Une nuit sur le mont Chauve de Moussorgski en nous

38 Les gorges du Darial, situées dans la région du Caucase, servent de décor au château de Tamara. Pour plus de détails sur le compositeur et cette œuvre en particulier, voir Campbell 2001. 
inspirant d'un autre tableau du peintre, exécuté dans le même style : Les gorges du Darial dans la nuit au clair de lune (1868) ${ }^{39}$.

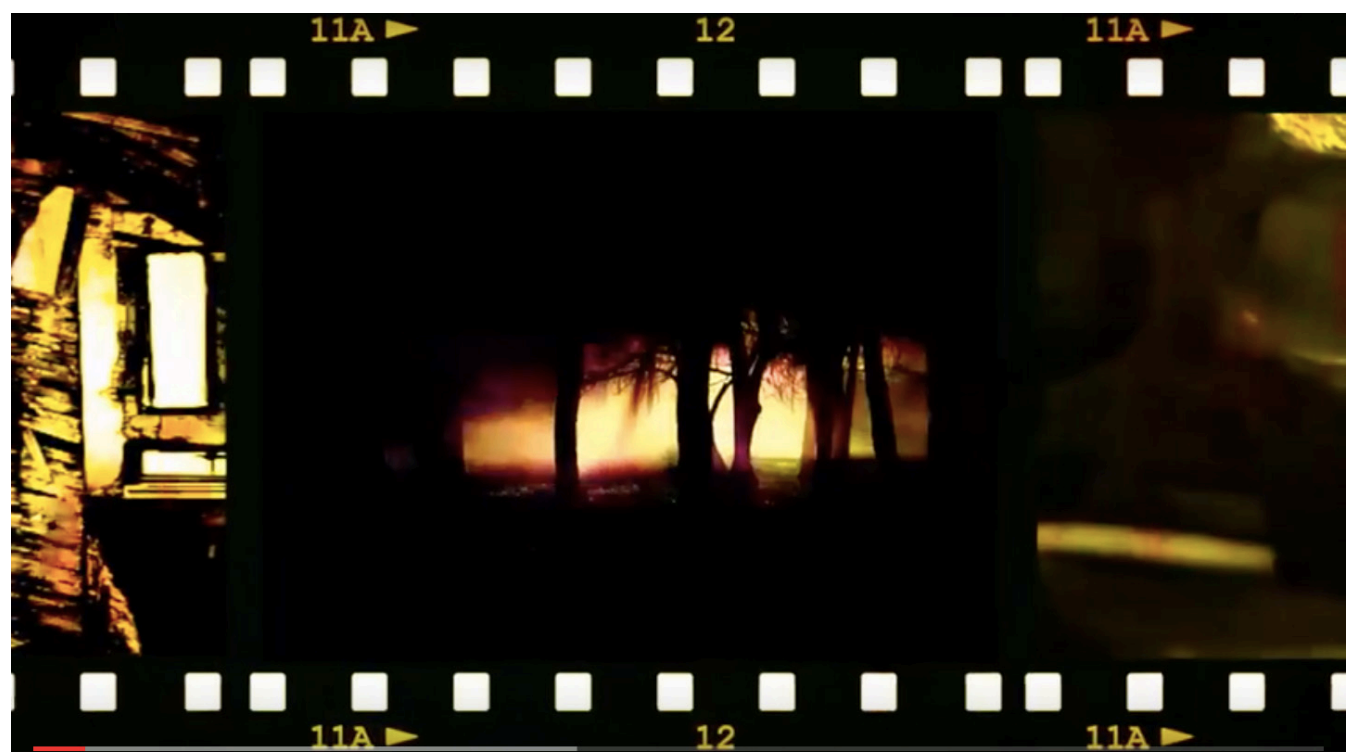

Figure 7: Capture d'écran de la capsule vidéo "Kent Nagano célèbre l'Halloween"; cinéma d'horreur (00:08).

En comparaison avec les précédentes capsules, les animations réalisées par Jason sont de qualité supérieure. Il y a non seulement la marche des balais pour L'apprenti sorcier, mais également les silhouettes de personnages mouvants : la mère et l'enfant pour illustrer La sorcière de midi de Dvoŕák ainsi que le voyageur égaré pour évoquer le récit de Tamara. Sur le plan sonore, notre esthétique s'est aussi raffinée. Il ne s'agissait plus, comme pour Pelléas, de faire entendre une musique de fond ; nous avons souhaité qu'il y ait une plus grande concordance avec les éléments visuels et narratifs ${ }^{40}$.

\section{La vie de Tchaikovski}

Les deux capsules sur Tchaikovski étaient placées sous le signe du changement. Devant la quantité de matière à couvrir, j'ai décidé, en concertation avec Jean-Claude Bernier et Michel Duchesneau, de tenter une version avec sous-titres. Le texte serait donc reproduit en bas de l'écran, groupe de phrases après groupe de phrases. Nous pouvions incorporer plus de contenus en un temps restreint, à condition que le rythme de défilement du texte soit assez élevé.

Nous passions non seulement à un modèle avec sous-titres, mais aussi à une capsule sur la vie d'un compositeur. Cette capsule ne serait pas diffusée dans le cadre d'un concert en particulier, mais à l'occasion du festival Tchaïkovski (6, 7 et 8 janvier 2016). Elle devait percussions, on imagine une dispute entre la mère et l'enfant; lorsque la section des cordes entame une longue descente chromatique, on imagine la voix tonitruante de la mère qui invoque la sorcière de midi. Ce sont ces deux effets musicaux que nous avons cherché à traduire en images (01:23 à 01:48). 
donc donner au public une vue d'ensemble. Pour ce faire, nous avons mentionné plusieurs de ses œuvres phares, notamment l'Ouverture Roméo et Juliette, le Concerto $n^{\circ} 1$ pour piano, Le lac des cygnes, l'Ouverture 1812 et la Symphonie $n^{\circ} 6^{41}$.

La musique de Tchaïkovski, caractérisée par de longues lignes mélodiques et une texture orchestrale très dense, pouvait difficilement être sectionnée en courts extraits musicaux. Hormis un fragment de 1'Ouverture 1812, nous avons donc choisi des passages plus longs de la Symphonie $n^{\circ}$ 6, laissant la musique se déployer amplement.

Le fragment de l'ouverture entendu est une citation musicale de La Marseillaise, 1'hymne national français, en référence aux troupes napoléoniennes qui, en 1812, sont entrées dans Moscou. Nous avons retenu cet extrait en partie parce qu'il s'agit d'un air bien connu du public. Visuellement, nous avons représenté Napoléon et son armée d'après un tableau de Victor Mazurovski (1859-1944), intitulé Napoléon quitte Moscou en $\mathrm{feu}^{42}$. En revanche, nous n'avons pas pris le temps d'inclure d'autres passages de l'œuvre qui empruntent, quant à eux, à la musique folklorique russe (thèmes issus de chansons populaires).

Pour pallier l'absence de sonorités typiquement russes dans cette capsule vidéo, le graphiste et moi avons cherché une identité visuelle associée - ou du moins associable - à la Russie. La fabrication artisanale des poupées russes et les clochers à bulbe d'oignon nous ont donné des premières pistes de réflexion. Notre choix s'est assez vite porté sur le style «folk art» dont quelques exemples m'avaient été présentés par Jason. Lui-même, dans sa propre démarche artistique, incorporait plusieurs éléments de ce style. Il m'a également montré des œuvres d'illustrateurs et illustratrices spécialisés dans cet art, notamment celles de Sanna Annukka.

À cela s'ajoutaient des photos de Tchaỉkovski, des portraits à différents âges de sa vie, des photos de lieux servant à le situer dans son époque et d'autres illustrations associées aux œuvres du compositeur. Pour cette capsule, le graphiste a également créé un nouveau type d'animations : le morphing, technique graphique visant à fondre progressivement une première image dans une autre. Celle-ci a été utilisée pour faire une transition entre l'illustration d'un piano à queue et celle d'un cygne qui glisse sur l'eau.

Extrait vidéo 5 : Capsule vidéo sur la vie de Tchaikovski. Voir la vidéo.

\footnotetext{
41 Avec les responsables de l'osm et Michel Duchesneau, la décision a été prise de faire une première capsule vidéo sur la vie du compositeur et une seconde sur sa Symphonie n ${ }^{\circ}$ 6, œuvre phare de Tchaïkovski et présentée dans le cadre du festival. L'intérêt était ici d'étaler équitablement le contenu musicologique sur deux capsules, l'une et l'autre étant complémentaires.

42 Date de réalisation du tableau inconnue. Toutefois, si l'on se réfère à l'année de naissance du peintre (1859), ce tableau aurait pu être exécuté vers 1912, à l'occasion du centenaire de la bataille victorieuse de la Russie contre Napoléon.
} 

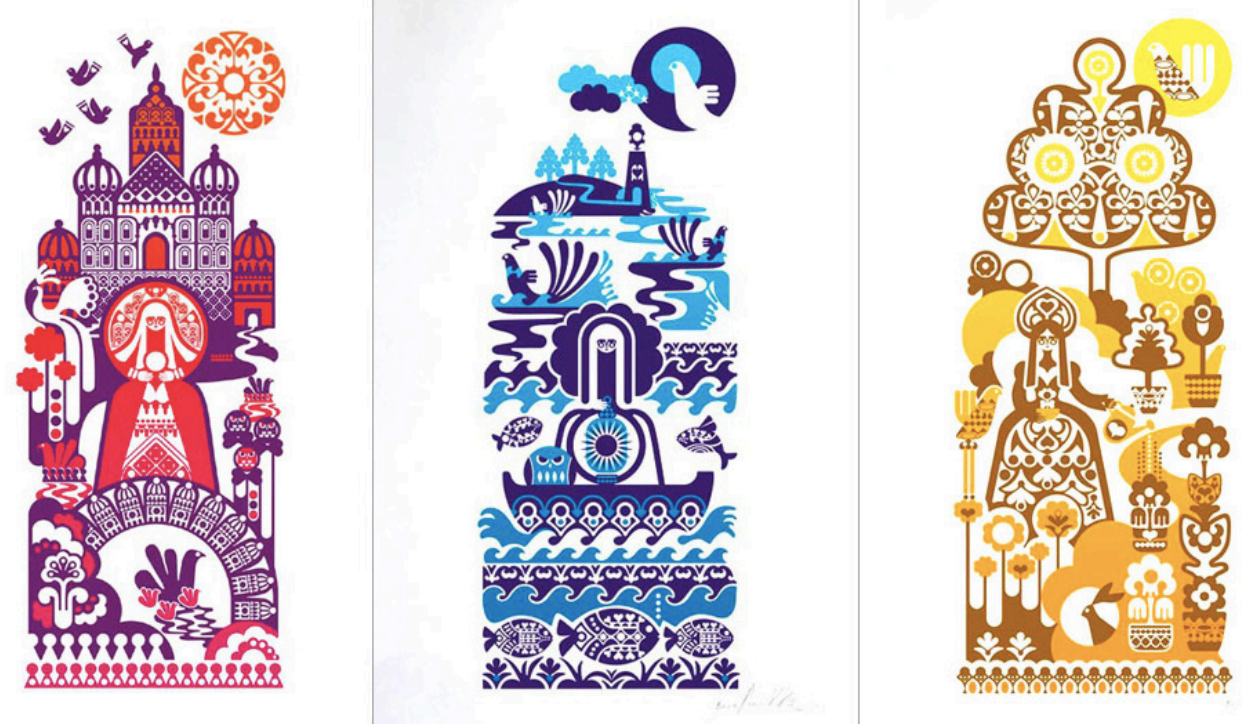

Figure 8: Série d'illustrations de Sanna Annuka ${ }^{43}$.

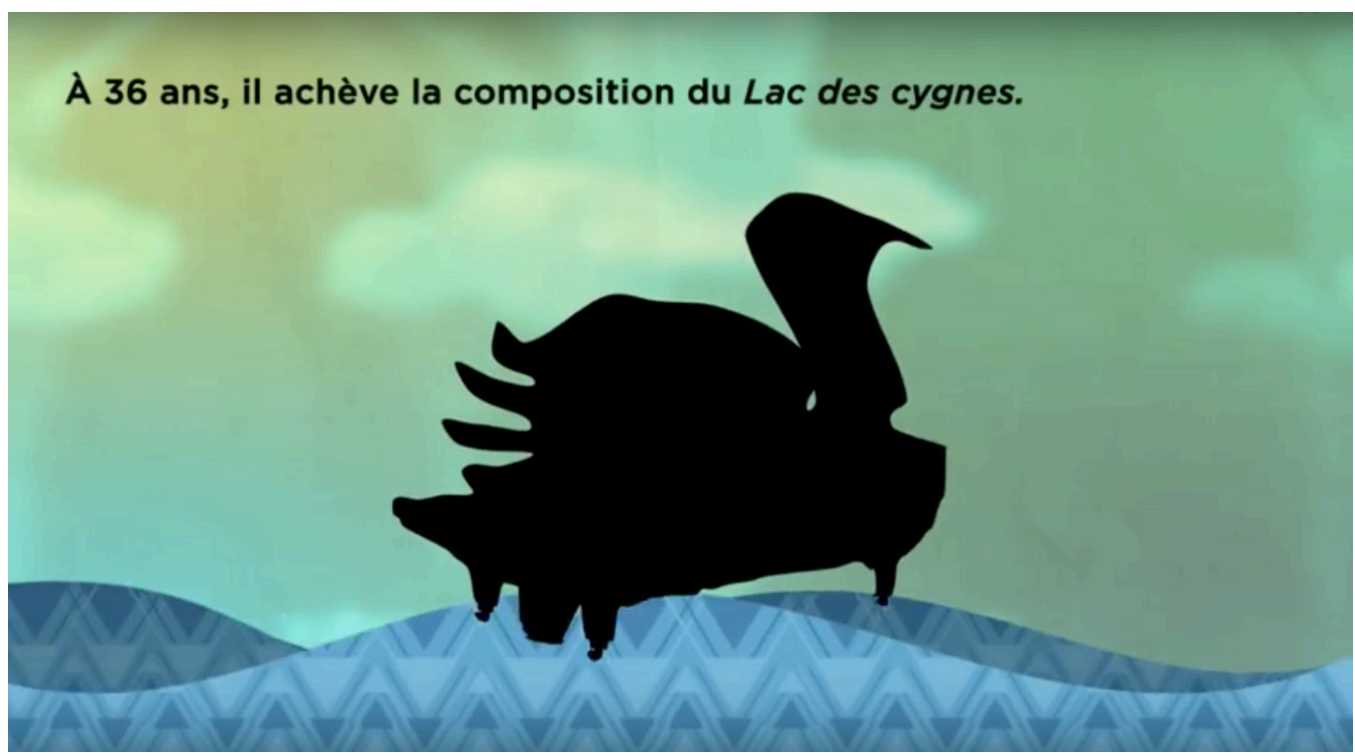

Figure 9: Capture d'écran de la capsule vidéo pour le festival Tchaïkovski; nouvelle animation (01:01).

43 Exemples trouvés sur le profil de l'utilisateur Matthew1clifford du site web PearlTrees (consulté le 6 janvier 2020). 
La Symphonie $\mathrm{n}^{\circ} 6$ de Tchaïkovski ${ }^{44}$

Il devait y avoir une concordance visuelle entre la conception graphique de la capsule sur la vie de Tchaïkovski et celle sur la Symphonie $n^{\circ}$ 6. Plusieurs éléments ont été écartés, notamment toute animation en référence aux autres œuvres de Tchaïkovski ainsi qu'aux autres compositeurs russes actifs à cette époque ${ }^{45}$. En revanche, afin de mieux comprendre le contexte de production de la Symphonie $n^{\circ}$ 6, un élément biographique a été ajouté. Nous voulions insister sur l'isolement de Tchaïkovski pendant la composition de l'œuvre, reclus dans sa maison en banlieue de Moscou.

Jason m'a parlé d'une nouvelle animation possible pour la partie analytique de la capsule. Dans un documentaire qu'il venait de visionner ${ }^{46}$, Jason avait été frappé par une séquence durant laquelle la caméra balaye l'écran d'une photo à l'autre, en avançant, et crée ainsi un effet de perspective. Le graphiste a voulu reprendre ce principe : il avait conçu plusieurs encadrés traitant de chaque mouvement de la symphonie. Le spectateur passait d'une information à l'autre, ce qui rendait les transitions très fluides. De plus, la clarté de la présentation assurait une meilleure compréhension de la structure de l'œuvre ainsi que des traits musicaux propres à chaque mouvement.

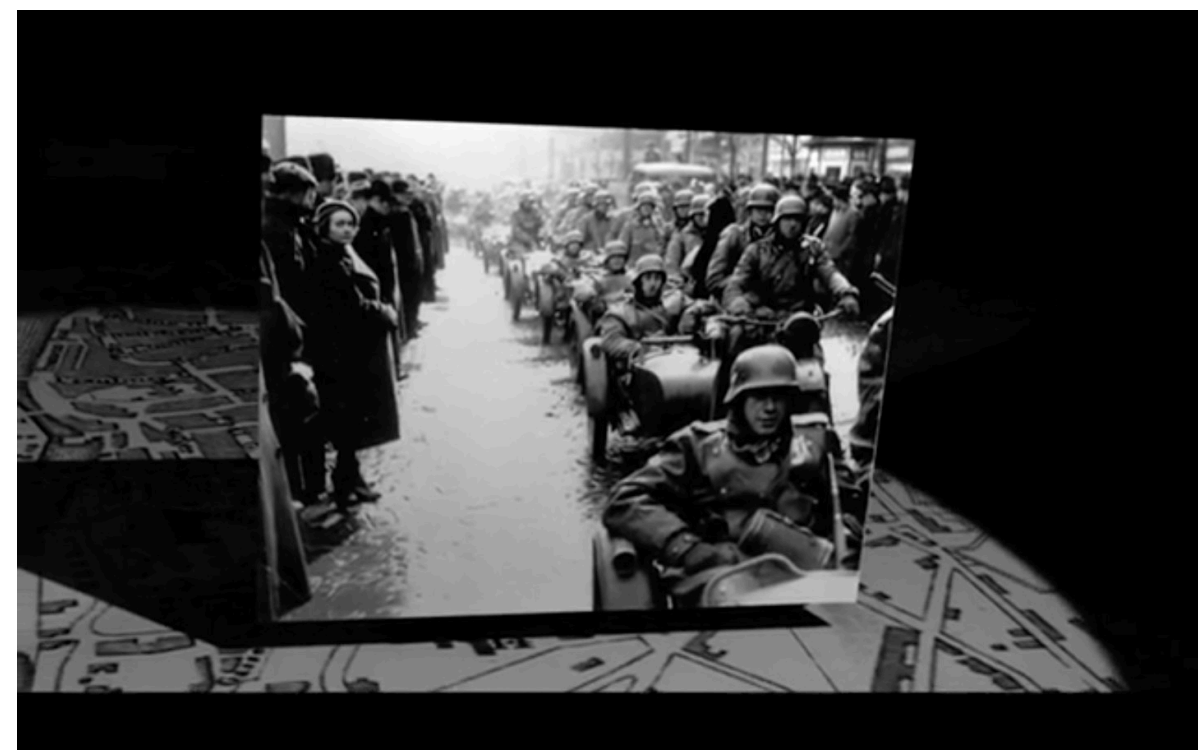

Figure 10: Capture d'écran du documentaire The Lady in Number 6 de Malcolm Clarke ; idée de transition (10:48).

44 Cette capsule vidéo n'a pas encore été diffusée officiellement sur les plateformes Facebook ou Youtube, que ce soit sur les comptes respectifs de l'OSM ou du DPMQ. Le graphiste ayant perdu malencontreusement une partie de ses données suite à un problème technique, nous n'avons pas nous-mêmes cette capsule vidéo en notre possession.

45 Nous faisons ici référence au groupe des Cinq : Balakirev, Borodin, Cui, Moussorgski et Rimski-Korsakov.

46 Ce documentaire, réalisé par Malcolm Clarke, s'intitule The Lady in Number 6. Music Saved My Life (2013). Il retrace la vie d'une pianiste juive ayant survécu à la Seconde Guerre mondiale. Disponible en ligne à l'adresse suivante : https://gloria.tv/video/Pf1cF3NbRNux4NiUUvBJYFDh4 (consulté le 6 janvier 2020). Un compte rendu rédigé par Laurence Manning est également disponible sur le site de la Revue musicale oICRM. 
Toutefois, cette nouveauté offrait un trop fort contraste avec le reste des animations de la capsule. À la demande de l'osm, nous sommes donc retournés à une esthétique plus conforme aux autres parties de la capsule.

En vue de la section consacrée à l'analyse musicale de l'œuvre, j'ai sélectionné des extraits musicaux et indiqué, sur une feuille de route, le minutage précis des enregistrements à utiliser. Dans le temps restreint qui nous était imparti, un ou deux éléments seulement de la partition étaient retenus pour chaque mouvement : des motifs ou thèmes prédominants (premier et troisième mouvements), un rythme singulier en $5 / 4$ (deuxième mouvement) ou encore le rappel d'un thème du premier mouvement dans le quatrième mouvement. Ainsi, l'animation visuelle s'est mise en adéquation avec le matériel musical, l'apparition de ces visuels répondant à la durée des extraits. À noter, par ailleurs, que la musique a servi de soutien expressif au texte, en particulier lorsque nous présentions les liens très étroits entre Tchaïkovski et son neveu Vladimir Davydov. Dans un premier temps, nous avons envisagé de rédiger un texte en vue d'une narration, mais avons finalement emprunté au modèle avec sous-titres, dans la lignée de la capsule consacrée au compositeur.

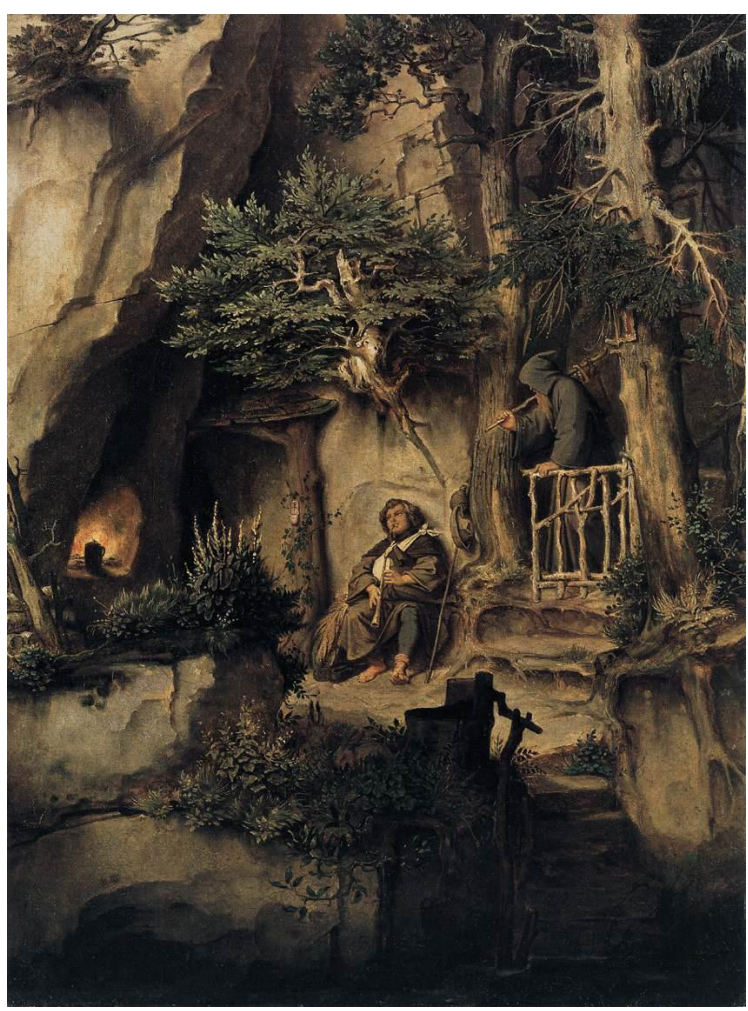

Figure 11 : Un ménestrel auprès d'un ermite (1846) de Moritz von Schwind.

La Symphonie no 1 de Mahler

Pour cette dernière capsule, nous avons privilégié une animation simple, qui se rapprochait davantage de la capsule sur l'ouverture de Guillaume Tell ou encore de celle sur la vie de Tchaïkovski : reproduction de photos d'époque, photos de Gustav Mahler et des lieux importants associés à la genèse et à la création de sa première symphonie, caricatures témoignant de l'accueil houleux qui a été réservé à cette œuvre. 
$\mathrm{Au}$ cours de mes recherches, j'avais pris connaissance d'une gravure de Moritz von Schwind (1804-1871) qui aurait inspiré Mahler pour le troisième mouvement de sa symphonie ${ }^{47}$. En découvrant les autres œuvres du peintre, j'ai constaté que plusieurs de ses toiles prenaient pour cadre la nature. Ces illustrations semblaient toutes désignées pour évoquer en images le programme sous-jacent de la Symphonie $n^{\circ} 1^{48}$. Plusieurs tableaux de la nature de Moritz von Schwind se sont ainsi retrouvés dans l'animation de la capsule.

Extrait vidéo 6: Capsule vidéo sur la Symphonie no 1 de Mahler. Voir la vidéo.

Dans le prototype de capsule sur l'ouverture de Guillaume Tell, nous avions également utilisé plusieurs toiles de paysages suisses - a fortiori par un maître de nationalité suisse comme Ferdinand Hodler. Dans le projet " Mahler », nous avons atteint, à notre avis, un meilleur équilibre entre contexte historique de composition, de création et analyse musicale de l'œuvre. Sur un total de 3 minutes et 54 secondes, $44,8 \%$ du temps a été consacré à cette partie analytique ( 1 minute et 45 secondes) et $38,5 \%$, à la présentation du contexte (1 minute et 30 secondes $)^{49}$. Il nous semblait important de restituer autant les ambitions du jeune Mahler, ses difficultés face aux critiques de l'époque, que les qualités musicales de la Symphonie $n^{\circ} 1$.

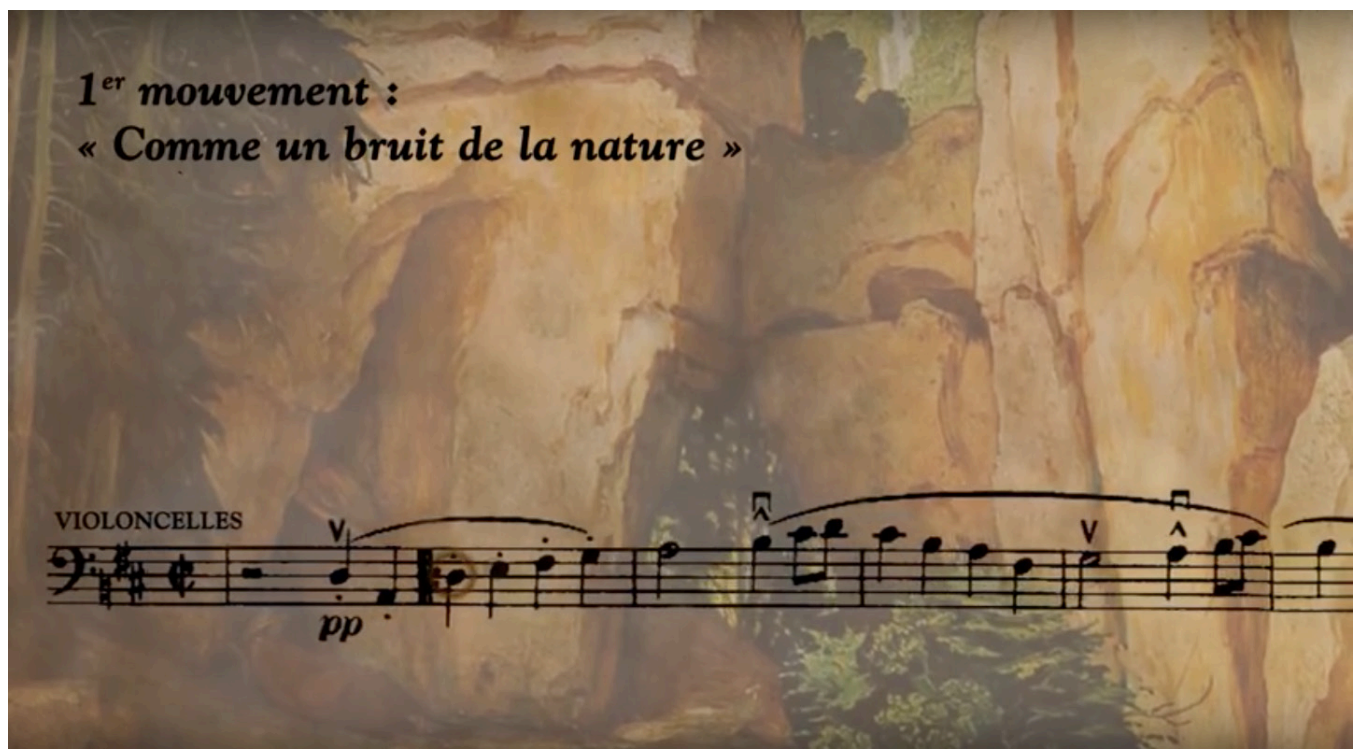

Figure 12 : Capture d'écran de la capsule vidéo sur la Symphonie n 1 de Mahler; animation de la partition (01:53).

47 Titre de la gravure : Wie die Tiere den Jäger begraben («Comment les animaux enterrent le chasseur »), produite en 1850 .

48 Le premier mouvement porte cette mention : "Wie ein Naturlaut» ("Comme un bruit de la nature »). À cela s'ajoutent des emprunts à la musique folklorique : une danse autrichienne (Ländler), que l'on entend dans le deuxième mouvement, et la mélodie de «Frère Jacques » en mineur, dans le troisième mouvement.

49 Les $16,7 \%$ restants correspondent aux séquences d'ouverture et de fermeture de la capsule (titre du début et générique de fin). À titre de comparaison, voici l'agencement des différentes parties de la capsule sur Les pins de Rome de Respighi - une œuvre également composée en quatre mouvements -, rapporté en pourcentage : ouverture et fermeture $(11,6 \%)$, contextualisation $(32,6 \%)$, partie consacrée à chaque mouvement de l'œuvre (55,8 \%). 


\section{RÉCEPTION DES CAPSULES VIDÉO ${ }^{50}$}

$\mathrm{Au}$ cours du présent article, nous nous sommes intéressés à plusieurs outils de médiation, anciens ou actuels, sans toutefois pouvoir démontrer concrètement leur efficacité ni leur écho auprès des auditeurs. Suite à la diffusion des capsules vidéo réalisées pour l'osm, la question demeure : quel effet ces outils ont-ils sur le public? Et qu'en pensent réellement les internautes?

L'administration de l'osm, par l'intermédiaire de Karine Ohayon Beaufils aux Communications, a accepté de nous communiquer certains chiffres de performance sur Facebook qui offrent de précieux éléments de réponse. Ces chiffres s'appliquent à quatre capsules, sur un total de six $^{51}$, incluant ceux du prototype sur l'ouverture de Guillaume Tell qui avait été diffusé le 19 février 2015, c'est-à-dire quatre mois avant le début de notre mandat au sein de l'administration.

Les premiers résultats dont nous disposons, concernant la capsule prototypique, indiquent 259 «j'aime », commentaires et partages répartis de la manière suivante :

- 192 «j'aime », dont 160 sur la publication (post) et 32 sur les partages d'internautes ;

- 34 commentaires, dont 25 sur la publication et 9 sur les partages ;

- 33 partages, seulement à partir de la publication elle-même et non à partir d'autres partages.

Nous reproduisons ici quelques exemples d'appréciation de la part d'internautes dont nous respectons l'anonymat :

Excellent document de présentation que j'aimerais bien utiliser pour aider dans l'appréciation de l'œuvre auprès de mes élèves. Serait-ce possible d'avoir un lien autre que FB [Facebook] pour les enfants ? - 19/02/2015, 17 h34.

Magnifique introduction à cette œuvre. À poursuivre. - 19/02/2015, $17 \mathrm{~h} 30$.

Excellente présentation ! Je sens que je vais la présenter à mon fils de 7 ans. 19/02/2015, $18 \mathrm{~h} 34$.

Excellent donne vraiment le goût d'y assister. - 20/02/2015, 6 h15.

Très réussi, visuel de qualité ; animations avec la partition en synchro avec la musique particulièrement appréciées. - 20/02/2015, 6h55.

Une idée originale dans le contexte qui permet une meilleure compréhension et appréciation de cette œuvre de Rossini qui a bercé mon enfance lorsque nous jouions aux cowboys et aux indiens et en faisant le ti-galop avions la mélodie en tête sans en connaître le compositeur ! Bravo ! - 20/02/2015, 9h21.

50 Cette section reprend une partie seulement des données recueillies sur Facebook, à propos de quatre capsules vidéo. Pour consulter l'ensemble des résultats obtenus, voir Bernard 2019, p. 306-313.

51 Nous avons perdu l'accès aux chiffres envoyés par l'osm sur notre ancienne boîte de réception institutionnelle (jbernar[a]osm.ca) et notamment les tout derniers chiffres sur la vidéo consacrée à la Symphonie $n^{o} 1$ de Mahler. De plus, nous ne connaissons pas en détail les chiffres concernant la capsule sur Les pins de Rome de Respighi (première capsule réalisée pendant le stage), mais nous savons, grâce à Karine Ohayon, qu'il s'agit de la vidéo ayant la moins bien performé par rapport aux quatre autres. 
Très bonne idée, il serait intéressant de voir davantage l'orchestre dans les capsules ! - 20/02/2015, $12 \mathrm{~h} 04$.

Excellent, cela permet de rendre les œuvres plus accessibles !! C'est un plus pour l'expérience du spectateur. - 20/02/2015, 13 h09.

Parmi ces témoignages, on trouve deux remarques d'internautes qui nous disent vouloir montrer la capsule à des enfants. C'est exactement ce à quoi nous aspirons en tant que développeurs d'outils de médiation numérique : rejoindre un public le plus large possible et pouvoir interpeller aussi bien les adultes que les jeunes et les enfants. À noter que sur l'ensemble des commentaires recueillis au sujet de la capsule consacrée à l'ouverture de Guillaume Tell, on en détecte un seul qui émette une certaine réserve. L'auteur de ce commentaire semble remettre en cause la pertinence de telles vidéos à caractère éducatif, préférant plutôt le rapport direct - non médié à l'œuvre musicale : "Étonnant ! J'appréciais cette ouverture sans en connaître les "dessous" expliqués dans le petit film, et je me demande encore si c'est bien utile de les connaître... » $(20 / 02 / 2015,18 \mathrm{~h} 02)^{52}$.

À titre de comparaison, la capsule vidéo sur Tchaïkovski a généré un total de 375 "j’aime », commentaires et partages. Voici ce que ces derniers résultats donnent dans le détail :

- 264 » j'aime », dont 141 sur la publication et 123 sur les partages d'internautes ;

- 26 commentaires, dont 3 sur la publication et 23 sur les partages ;

- 85 partages, dont 83 à partir de la publication originale et 2 à partir d'autres partages.

Ces chiffres soulignent une tendance déjà observée lors de la diffusion de la capsule consacrée au concert d'Halloween. Les internautes « aiment » et partagent les vidéos en grand nombre, mais ils commentent moins ; ils interagissent donc moins verbalement. Lorsqu'une campagne médiatique importante est organisée en amont, la publication contenant la vidéo sera plutôt perçue comme un élément de ce dispositif, servant à publiciser le concert à venir, permettant aussi à l'utilisateur de Facebook de partager l'information auprès de ses amis, et moins comme un outil de médiation. L'intérêt pour la valeur éducative de la capsule baisse et, avec lui, l'intérêt de s'exprimer sur son contenu. En témoignent quelques commentaires, retrouvés au bas de la capsule sur le concert d'Halloween :

52 Afin de répondre, entre autres, à la question fondamentale, voire existentielle, de savoir si les outils de médiation favorisent ou non l'appréciation des œuvres musicales, nous avons réalisé une enquête auprès du public de l'osm, au cours d'une série de trois concerts à la Maison symphonique (octobre 2017). Les résultats de cette enquête montrent qu'une nette majorité de répondants $(82,6 \%)$ considère que les notes de programme ont un effet positif sur la qualité d'écoute de la musique (écoute plus attentive), contre 9,4\% des répondants qui ne le pensent pas ( $8 \%$, sans opinion). Pour plus de détails sur l'ensemble des résultats de l'enquête, voir Bernard, 2019, p. 219-251. 
Trop hâte ! - 16/10/2015, 9h53.

[En «taguant» une amie] Ça serait cool hein ? - 16/10/2015, $10 \mathrm{~h} 07$.

Tellement * $0 *$. $17 / 10 / 2015,14 \mathrm{~h} 41$.

Billet acheté !! - 16/10/2015, 10h58.

Oh yeah! Tellement [hâte] d'y aller ! Et en excellente compagnie qui plus est !! Jan aura finalement sa danse macabre ! Il en frétille d'excitation! Ahahah. 16/10/2015, 20h00.

Billet acheté, pub génial[e]. - 17110/2015, 5h22.

Oui, nous y serons à cette soirée...nous sommes heureux de pouvoir vivre cet évènement de mystère et de suspense... - 24/10/2015, $15 \mathrm{~h} 36$.

Superbe idée...diabolique !!! - 25/10/2015, 7h45.

La plupart des commentaires laissés par les internautes peuvent être résumés ainsi : mentions du concert-évènement, appels à assister à cette soirée, signalements de l'intérêt pour ce type de répertoire - intérêt renforcé par les interactions d'utilisateurs et amis Facebook -, mais de rares allusions à la capsule elle-même. Dans la série d'exemples mis en exergue ci-dessus, on relève un commentaire pouvant prêter à interprétation - en s'exclamant « Superbe idée... diabolique !!! », l'internaute parle-t-il du concert ou de la vidéo ? - et un seul commentaire explicite sur la vidéo ; celle-ci est jugée comme une " pub géniale ». Ici, c'est la valeur marketing, commerciale, et non la valeur éducative que l'on attribue à la capsule ; preuve qu'un tel outil de médiation peut aussi bien être perçu, sinon plus, comme un moyen de promotion, intégré à une campagne marketing, que comme un instrument répondant au volet " éducation » d'un orchestre.

\section{CONCLUSION}

Nous aurions eu tort de réduire ce projet de capsules vidéo à une simple réitération de ce que sont déjà les notes de programme. L'occasion nous a été offerte de remettre au goût du jour certains éléments qui avaient été abandonnés dans le format papier, comme les extraits de partition ou les réductions de thèmes musicaux pour piano. Plus qu'une béquille pour des auditeurs qui ne lisent pas la musique, le défilement d'extraits musicaux à l'écran devait pouvoir servir de guide audio-visuel ; un guide qui faciliterait la lecture de partition grâce notamment à l'animation graphique d'un motif ou d'un thème musical, ainsi rehaussé par des effets de couleurs. De plus, les capsules vidéo nous ont permis d'explorer les possibilités d'un nouveau mode de communication, capable d'interpeller plus directement l'auditeur et d'associer visuellement la musique à des références issues de la culture populaire, susceptibles d'être comprises par l'auditeur. Dès le début de la capsule sur Les pins de Rome de Respighi, nous avons fait allusion à des films connus du grand public (La guerre des étoiles, Superman) afin d'attirer l'attention des internautes sur les qualités sonores de l'œuvre qui rappellent de nombreuses musiques composées pour le cinéma.

Dans cet article, nous avons mis en lumière certains modèles de médiation qui ont été, pour nous, des sources d'inspiration et de motivation. Nous avons souhaité ensuite documenter les différentes étapes et les choix nécessaires à la réalisation de chacun de nos projets de capsules vidéo. Cette expérience a été riche d'enseignements. 
Dans la perspective de projets futurs, il est notamment à prévoir que le musicologue se heurte, d'une part, aux attentes non comblées de l'institution et, d'autre part, à l'essoufflement de l'intérêt du public pour ce type d'intervention, l'effet de nouveauté se diluant assez rapidement au fil d'une série. Pour remédier à ces deux facteurs de risque, il est essentiel de trouver préalablement un terrain d'entente entre les différents acteurs, c'est-à-dire de mettre en accord les motivations que partagent le musicologue et les autres intervenants avec celles de l'institution musicale mandataire. Il s'agit aussi de déterminer le rôle précis que nous voulons collectivement faire jouer aux outils de médiation numériques, un rôle parfois ambivalent entre promotion d'un concert (vision à court terme) et développement de public (vision à long terme).

\section{BIBLIOGRAPHIE}

Agawu, Kofi (2009), « La part de l'oralité en analyse musicale », dans Rémy Campos et Nicolas Donin (dir.), Analyse musicale, une pratique et son histoire, Genève, Haute École de Musique de Genève, p. 123-130.

Bashford, Christina (2003), "Not Just "G." Towards a History of the Programme Note ", dans Michael Musgrave (dir.), George Grove, Music and Victorian Culture, Basingstoke, Palgrave Macmillan, p. 115-142.

Bashford, Christina (2007), The Pursuit of High Culture. John Ella and Chamber Music in Victorian London, Woodbridge, Boydell Press.

Béchemin, Cyril, Géraldine Cohen, et Olivier Rampnoux (2016), "Le Livre parle à ses lecteurs. Exploration des implications dans le processus de conception ", Revue de l'Enssib, $\mathrm{n}^{\circ} 4$, http://bbf.enssib.fr/revue-enssib/consulter/revue-2016-04-007, consulté 2 février 2018.

Bernard, Justin (2019), Notes de programme. Une histoire, des pratiques et de nouveaux usages numériques, thèse de doctorat, Université de Montréal.

Bernstein, Leonard (1959), The Joy of Music, New York, Simon and Shuster.

Campbell, Stuart (2001), " Balakirev, Mily Alekseyevich », dans Oxford Music Online, https://doi.org/10.1093/gmo/9781561592630.article.40685, consulté le 22 juillet 2018.

Debussy, Claude (1987), Monsieur Croche et autres écrits, Paris, Gallimard.

Gottlieb, Jack (2010), Working with Leonard Bernstein: a Memoir, Milwaukee, Amadeus Press.

Igonet, Aurélie (2005), "Une architecture du son dans la communication multimédiatée », dans Rémi Adjiman et Bruno Cailler (dir.), Une architecture du son, Paris, L'Harmattan, p. 218-253.

Maisonneuve, Sophie (2009), "L'art d'écouter la musique. Les commentaires discographiques des années 1910 à 1950 : une genèse et paradigme d'une nouvelle pratique analytique ", dans Rémy Campos et Nicolas Donin (dir.), Analyse musicale, une pratique et son histoire, Genève, Haute École de Musique de Genève, p. 141-278.

Ross, Alex (2008), The Rest is Noise. Listening to the Twentieth Century, New York, Picador.

\section{SitOGRAPHIE}

Capsule vidéo sur l'ouverture de Guillaume Tell de Rossini, vidéo YouTube, https://www.youtube. com/watch? $\mathrm{v}=04 \mathrm{vy} 4$ pojpHA, consulté le 8 janvier 2020.

Capsule vidéo sur Les Pins de Rome de Respighi, vidéo YouTube, https://www.youtube.com/ watch?v=p9JFfysFaKA, consulté le 8 janvier 2020. 
Capsule vidéo sur Pelléas et Mélisande de Debussy, vidéo YouTube, https://www.youtube.com/ watch?v= WZnUxXw8rw, consulté le 8 janvier 2020.

Capsule vidéo préparée pour le concert "Kent Nagano célèbre l'Halloween ", vidéo YouTube, https://www.youtube.com/watch?v=slya5SYb5DE, consulté le 8 janvier 2020.

Capsule vidéo préparée pour le festival « Tchaïkovski », vidéo YouTube, https://www.youtube.com/ watch?v=dGBnbq9YLzk, consulté le 8 janvier 2020.

Capsule vidéo sur la Symphonie $n^{\circ} 1$ de Mahler, vidéo YouTube, https://www.youtube.com/ watch?v=70oceV4kThM, consulté le 8 janvier 2020.

Euvres de l'illustratrice Sanna Annuka, PearlTrees, http://www.pearltrees.com/matthew1clifford/ sanna-annukka/id12469469, consulté le 8 janvier 2020.

Documentaire intitulé The Lady in Number 6 (2014), Gloria.tv, https://gloria.tv/video/ Pf1cF3NbRNux4NiUUvBJYFDh4, consulté le 8 janvier 2020.

Collection " Le Petit Ménestrel », Bibliothèque nationale de France, http://data.bnf.fr/13882180/le petit menestrel, consulté le 8 janvier 2020.

Collection "Le Petit Ménestrel », Discogs, https://www.discogs.com/fr/label/147672-Le-PetitMénestrel?page $=1$, consulté le 8 janvier 2020.

Projet de "Partenariat sur les publics de la musique " ( $\left.\mathrm{P}^{2} \mathrm{M}\right)$, OICRM, http://p2m.oicrm.org/p2mrecherche/presentation-du-projet, consulté le 8 janvier 2020.

Série de concerts, Reid Orchestra, www.reidconcerts.music.ed.ac.uk, consulté le 8 janvier 2020

Équipe de recherche du DPMQ, OICRM, http://dpmq.oicrm.org, consulté le 8 janvier 2020.

Vidéo promotionnelle de la saison 2015-2016 de l'osm, vidéo YouTube, https://www.youtube.com/ watch?v=70nz77-QK34, consulté le 8 janvier 2020.

\section{INSTITUTIONS MUSICALES RÉPUTÉES POUR LEUR OFFRE NUMÉRIQUE PÉDAGOGIQUE}

Aux États-Unis :

Philadelphia Orchestra (application LiveNote), https://www.philorch.org/concert/livenote-2019-20\#, consulté le 8 janvier 2020.

San Francisco Symphony (deux projets: Program Note Podcastset Keeping Score), http:/ /www.sfsymphony. org/Watch-Listen-Learn, http://www.keepingscore.org, consulté le 8 janvier 2020.

En Europe :

Orchestre national d'Île-de-France (deux web-séries : Allez, raconte Camille et MarmitOndif), http://www.dailymotion.com/playlist/x3gch2 ONdIF allez-raconte-camille/1\#video=x37z4fk, https://www.youtube.com/playlist?list=PLzNGb01kQaoZ9IF-pSZIOixlaXW7F0dZV, consulté le 8 janvier 2020.

Philharmonia Orchestra (guides d'écoute), http://www.philharmonia.co.uk/explore/films/ listening guides, consulté le 8 janvier 2020. 
ANNEXE : Exemples de textes préparatoires pour la réalisation des capsules vidéo.

a) «Pélléas_narration_version 9 (version courte)» : document ayant servi à l'enregistrement de la narration pour la capsule sur Pelléas et Mélisande de Debussy

Dans Pelléas et Mélisande, Claude Debussy rompt avec la tradition de l'opéra du $\mathrm{XIX}^{\mathrm{e}}$ siècle pour libérer le public des conventions musicales. Finis les grands airs et le lyrisme : les lignes vocales imitent désormais la voix parlée.

C'est là d'où vient le reproche que l'on fait à mon soi-disant parti-pris de déclamation monotone où jamais rien n'apparaît de mélodique. D'abord, c'est faux : les sentiments d'un personnage ne peuvent s'exprimer continuellement d'une façon mélodique.

Pelléas et Mélisande, c'est d'abord une pièce de théâtre signée Maurice Maeterlinck qui allait aussi inspirer d'autres compositeurs. Pour Debussy et Maeterlinck, tout est dans l'art de suggérer, d'évoquer des images, de dire les choses à-moitié, à la fois en paroles et en musique. De ce point de vue, ils appartiennent au mouvement symboliste.

Les personnages principaux sont comme deux enfants qui vivent leur premier amour. Un amour qui sera avoué à la fin de l'œuvre seulement. Leur histoire est innocente, mais elle provoquera la jalousie de Golaud, demi-frère de Pelléas et époux forcé de Mélisande.

Créé en 1902, Pelléas et Mélisande est l'unique opéra de Debussy, son œuvre sans doute la plus importante. Elle établit un langage musical résolument tourné vers la modernité : abandon du système tonal tel que l'employaient les compositeurs romantiques, exploration de nouvelles sonorités. Rien n'est véritablement affirmé. Tout reste à l'état d'impressions sonores.

Deux idées sont omniprésentes dans cet opéra : l'eau et la chevelure de Mélisande, toutes deux liées par une sensation d'ondulation.

Plusieurs scènes se déroulent autour d'une fontaine mystérieuse, dont on peut d'ailleurs entendre une imitation musicale.

$\mathrm{Au}$ début du troisième acte, Mélisande évoque sa longue chevelure dans une mélodie qui rappelle un chant grégorien.

Dans l'histoire de la musique classique, peu d'œuvres auront suscité autant de passions que Pelléas et Mélisande ! À commencer par le violent affrontement entre Debussy et Maeterlinck au moment des premières répétitions. Sujet de la discorde : le choix de l'interprète pour le rôle de Mélisande. C'est finalement la soprano écossaise Mary Garden et non Georgette Leblanc, actrice et maîtresse de Maeterlinck, qui sera l'élue. Furieux, Maeterlinck se lance alors dans un combat personnel contre Debussy, envisageant même de le provoquer en duel : «Je suis obligé d'espérer que ce sera une faillite retentissante et rapide».

L'opéra fait scandale. Il devient le théâtre de nouveaux affrontements entre le public conservateur et l'Avant-Garde musicale. Heureusement, la résistance s'essouffle peu 
à peu et l'œuvre connaît un succès croissant, ce qui vaudra à Debussy une renommée internationale.

b) Feuille de route en vue de la capsule vidéo sur la Symphonie $n^{\circ} 6$ de Tchaïkovski, avec minutage des extraits audio (didascalies marquées en vert)

\section{Première section : composition de l'œuvre et vie du compositeur}

$$
\text { (Piste } 4 \text { [4 mouvement]. 00:00) }
$$

Piotr Ilitch Tchaïkovski (1840-1893)

Symphonie $n^{\circ} 6$ en si mineur, "Pathétique»

1893

L'œuvre ultime de Tchaïkovski.

Ultime dans tous les sens du terme...

Tchaikovski meurt seulement huit jours après la première qu'il avait dirigée lui-même à Saint-Petersbourg, le 28 octobre 1893.

Causée par le choléra, sa mort foudroyante marque profondément les esprits.

Quelques semaines plus tard, la deuxième interprétation de l'œuvre sera dirigée par Eduard Nápravník lors d'un concert-hommage exceptionnel.

Selon Tchaïkovski, la Symphonie "Pathétique » est son œuvre la plus aboutie. « Je la considère comme la meilleure - et surtout la plus sincère - de toutes mes œuvres. Je l'aime comme jamais je n'ai aimé mes enfants musicaux. » (lettre à son neveu, Vladimir Davydov, 14 août 1893)

Le compositeur avait écrit sa Sixième symphonie avec un programme en tête, une histoire qui lui servait d'inspiration.

«Ce programme est profondément empreint de sentiments subjectifs, et il m'est arrivé à maintes reprises, en le composant mentalement, de verser beaucoup de larmes. » (lettre à Vladimir Davydov, 11 février 1893)

Malheureusement, Tchaïkovski n'a laissé aucune trace de ce programme. La véritable histoire sous-jacente semble être celle de sa propre existence. 
En février et au cours de l'été 1893 ,

Tchaikovski poursuit la composition de sa symphonie, reclus dans sa maison de Klin, près de Moscou.

Il souffre de l'éloignement de ses proches qui demeurent à Saint-Pétersbourg, notamment son neveu Vladimir Davydov à qui il dédie la "Pathétique "

$$
(01: 21.2)
$$

\section{Deuxième section : analyse musicale de l'œuvre}

\section{Allegro non troppo}

(Piste 1 [1 $1^{\text {er }}$ mouvement]. De 01:55 à 02:05)

Le premier mouvement de la «Pathétique » est marqué par un motif joué aux violons.

Animation de partition

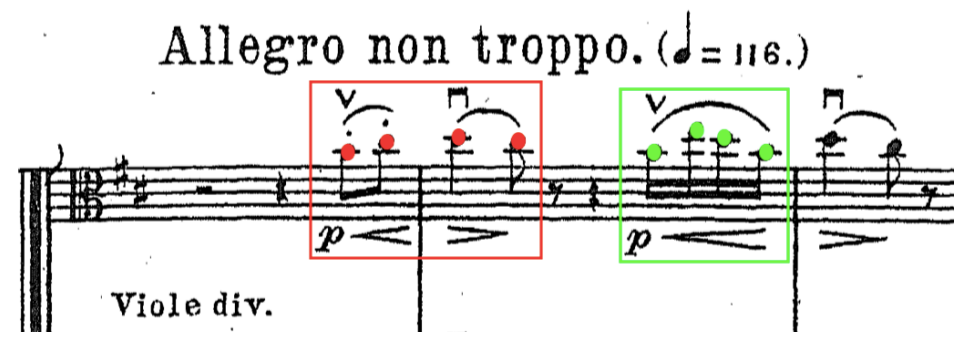

page 5 de la partition

Mesures suivantes avec partition, de 02:06 à 02:14.3

(12:27 à 12:34.4)

Lorsque le tempo s'accélère, ce motif récurrent génère une forte tension dramatique entre la section des cordes et des cuivres.

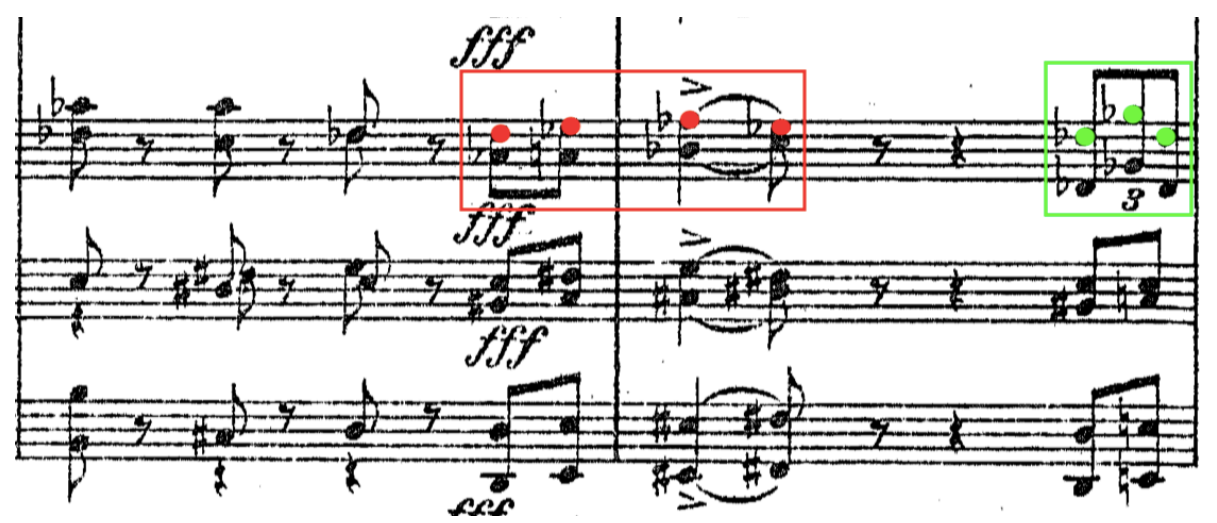

page 56 de la partition 


\section{Allegro con grazia}

(Piste 2 [2e mouvement]. De 01:11 à 01:28.2)

Le deuxième mouvement fait entendre un rythme insolite à cinq temps... Son ton léger contraste avec le début de l'œuvre.

\section{Musique sans partition}

\section{Allegro molto vivace}

\section{(Piste 3 [3 $3^{\mathrm{e}}$ mouvement]. De 05:59.6 à 06:05)}

Le troisième mouvement est porté par un élan énergique.

Le thème prédominant revient de manière presque obsessionnelle.

Animation de partition

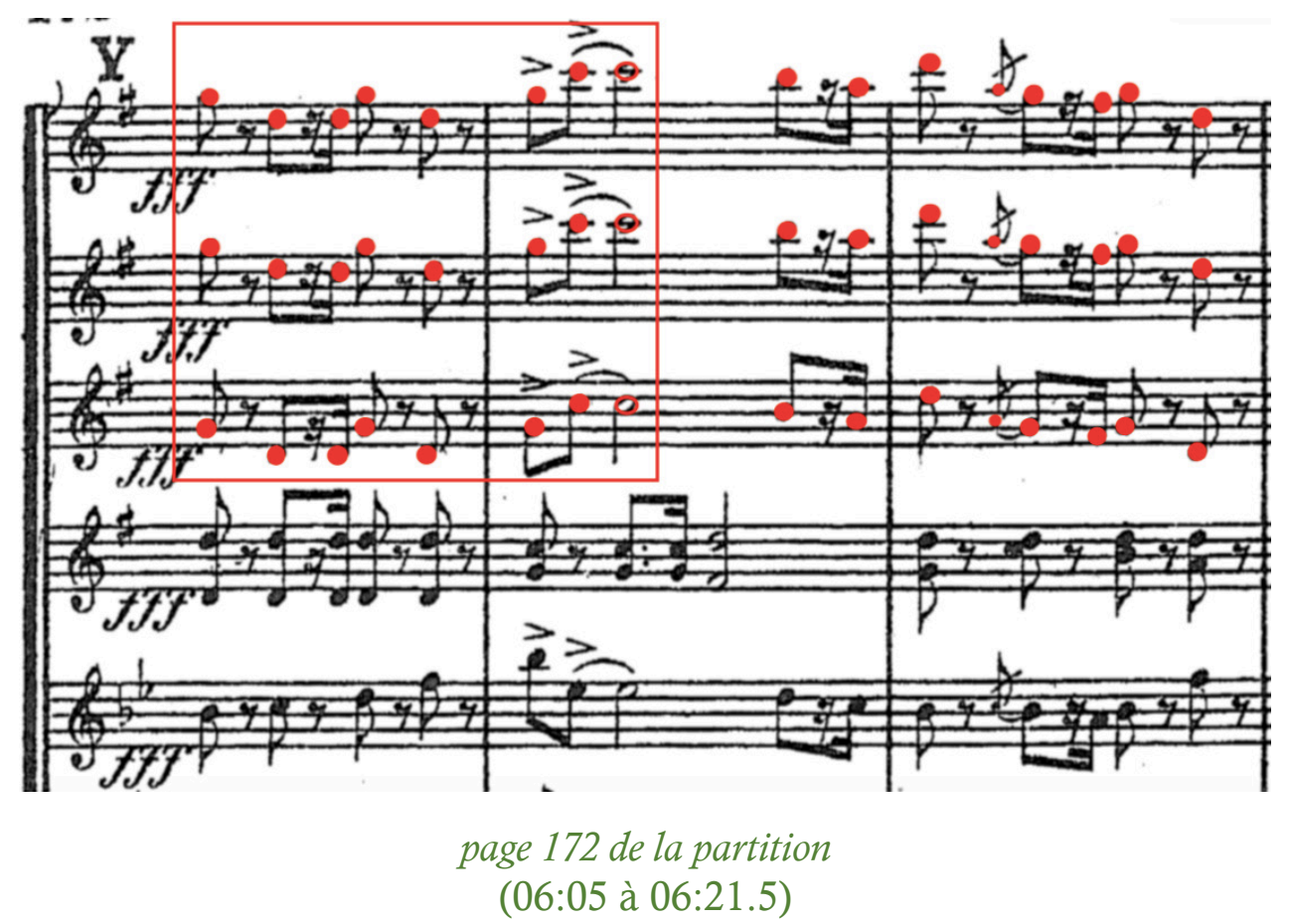

\section{Adagio lamentoso}

(Piste 4. 05:27 à 05:48.9)

Le quatrième mouvement est un Adagio lamentoso, tempo inhabituellement lent pour conclure une symphonie. D'emblée, le thème musical rappelle celui du premier mouvement, chargé, cette fois, d'une profonde tristesse.

page 210 de la partition, numéro $G$.

Détail de partition avec animation (05:41 à 05:48.9) 
Troisième section (esthétique comparable à la première)

(05:49 à 06:05, générique de fin inclus)

Cette symphonie est aujourd'hui considérée comme le testament musical de Tchaïkovski, une œuvre emblématique de son style et indissociable de sa vie personnelle. 